\title{
EVOLUTION OF ELASTIC THIN FILMS WITH CURVATURE REGULARIZATION VIA MINIMIZING MOVEMENTS
}

\author{
PAOLO PIOVANO
}

\begin{abstract}
The evolution equation, with curvature regularization, that models the motion of a two-dimensional thin film by evaporation-condensation on a rigid substrate is considered. The film is strained due to the mismatch between the crystalline lattices of the two materials. Here, short time existence, uniqueness and regularity of the solution are established using De Giorgi's minimizing movements to exploit the $L^{2}$-gradient flow structure of the equation. This seems to be the first analytical result for the evaporationcondensation case in the presence of elasticity.
\end{abstract}

\section{INTRODUCTION}

In this paper we study the morphologic evolution of an anisotropic epitaxial film deposited on a rigid substrate, with the film strained due to a mismatch between the crystalline lattices of the two materials. We consider the evaporation-condensation case and neglect surface diffusion, with the profile of the film being modeled as a grain-vapor interface with the vapor being considered as a reservoir that interacts with the profile of the film only through the evaporation-condensation process (see [20, Section 19]). We essentially follow the approach that is used in [19] for the surface diffusion case, and just as in [19] we restrict our attention to the two-dimensional model or, in other words, to a three-dimensional epitaxially strained film with identical vertical cross-sections.

One of the earliest theories for the evolution of an interface $\Gamma$ between two phases is due to Mullins (see [33, 34]), who derived the equations that describe the planar motion of isotropic grain boundaries by evaporation-condensation and by surface diffusion. Up to a rescaling, the equations are the motion by mean curvature and the motion by surface Laplacian of mean curvature, i.e.,

$$
V=k \quad \text { and } \quad V=-k_{\sigma \sigma} \text { on } \Gamma,
$$

respectively, where $V$ is the normal velocity, $k$ is the curvature of the evolving interface and $(\cdot)_{\sigma}$ is the tangential derivative along the interface. There is a large body of literature devoted to the study of these equations. In particular, a generalization of Mullins's models includes anisotropy (see [20, Section 19.7]). Precisely, the anisotropic surface energy

Date: May 14, 2012.

Key words and phrases. minimizing movements, gradient flow, evolution, motion by mean curvature, thin film, anisotropy, elasticity, epitaxy, evaporation-condensation, grain boundaries.

2010 Mathematics Subject Classification: 35K30, 49J10, 74K35, 74N20. 
functional is

$$
\int_{\Gamma} g(\theta) \mathrm{d} \mathcal{H}^{1}
$$

where the surface energy density $g$ is evaluated at the angle $\theta$ that the surface normal vector $\nu$ forms with the $x$-axis and $\mathcal{H}^{1}$ denotes the one-dimensional measure on the evolving interface $\Gamma$. In particular, in $[23$, Section 8$]$ and $[7,25]$ it is shown that the equation for the evaporation-condensation case becomes

$$
\beta V=\left(g_{\theta \theta}+g\right) k-U \text { on } \Gamma,
$$

where $U$ is a constant and the kinetic coefficient $\beta$ is a material function associated with the attachment kinetics of the atoms at the interface. We assume the kinetic coefficient to be constant and so, up to a rescaling, we take $\beta \equiv 1$.

Locally, the interface may be described as the graph of a one-dimensional function. In the context of a thin film over a flat substrate, we set the $x$-axis on the substrate upper boundary and describe the thickness of the film by means of a profile function $h:(0, b) \times[0, T] \rightarrow[0, \infty)$ for a positive length $b$ and a positive time $T$. In this way, the graph of $h$ represents the evolving profile $\Gamma_{h}$ of the film. We adopt the sign convention that the normal vector $\nu$ points outward from the region $\Omega_{h}$ occupied by the film and $k$ is negative when the profile is concave. Note that the normal velocity parametrized by the profile function $h$ is given by

$$
V=\frac{1}{J} h_{t}, \quad \text { where } \quad J:=\sqrt{1+\left|h_{x}\right|^{2}}
$$

and we denote by $h_{x}$ and $h_{t}$ the derivatives with respect to the first and the second component, respectively.

In $[7,23]$, the constant $U$ is included in (1.3) to represent the difference in bulk energies between the phases. As already mentioned in [23, Remark 3.1], the theory can be extended to account for deformation (see also $[20,26]$ ). Indeed, the inclusion of deformation is very important to model epitaxy because the difference in lattice parameters between the film and the substrate can induce large stresses in the film. In order to release the resulting elastic energy, the atoms in the film move and reorganize themselves in more convenient configurations. In analogy with $[10,18,21]$ and with the surface diffusion case (see [19]), we work in the context of the elasticity theory for small deformations. Hence, fixing a time in $[0, T]$, the linearized strain is represented by $E(u)=\frac{1}{2}\left(\nabla u+\nabla^{T} u\right)$, where $u$ defined on $\Omega_{h}$ denotes the planar displacement of the bulk material that is assumed to be in (quasistatic) equilibrium, and the bulk elastic energy is

$$
\int_{\Omega_{h}} W(E(u)) \mathrm{d} z
$$

where the elastic energy density $W: \mathbb{M}_{\text {sym }}^{2 \times 2} \rightarrow[0, \infty)$ is defined by

$$
W(A):=\frac{1}{2} \mathbb{C} A: A,
$$


for a positive definite fourth-order tensor $\mathbb{C}$. Furthermore, we model the displacement of the film atoms at the interface with the substrate using the Dirichlet boundary condition $u(x, 0)=\left(e_{0} x, 0\right)$, where the constant $e_{0}>0$ measures the mismatch between the crystalline lattices. Moreover, the migration of atoms can eventually result in the formation of surface patters on the profile of the film, such as undulations, material agglomerates or isolated islands. However, these non-flat configurations have a cost in terms of surface energy which is roughly proportional to the area of the profile of the film (see (2.3) below). Therefore, the evolution of the film profile is the result of the competition between the bulk elastic energy and the surface energy of the film, and (1.3) becomes

$$
V=\left(g_{\theta \theta}+g\right) k-W(E(u)) \text { on } \Gamma_{h},
$$

while the corresponding equation in the case of surface diffusion is

$$
V=\left(-\left(g_{\theta \theta}+g\right) k+W(E(u))\right)_{\sigma \sigma} \text { on } \Gamma_{h},
$$

where $W(E(u))$ is defined for each $t \in[0, T]$ as the trace of $W(E(u(\cdot, t)))$ on $\Gamma_{h(\cdot, t)}$ and $u(\cdot, t)$ is the elastic equilibrium corresponding to $h(\cdot, t)$.

These evolution equations exhibit different behaviors with respect to the sign of the interfacial stiffness $f:=g_{\theta \theta}+g$. In fact, the equations are parabolic on any angle interval in which $f$ is strictly positive. In this case, (1.5) has been extensively studied and it behaves similarly to $V=k$ (see, e.g., $[5,6,25]$ ). Those angle intervals in which $f$ is negative are relevant from the materials science viewpoint. In this range, (1.5) is backward parabolic and unstable and so, in order to analyze its behavior, we consider a higher order perturbation. The idea consists in allowing for a dependence on curvature of the surface energy density $g$ in order to penalize surface patterns with large curvature, such as sharp corners (see $[35,38]$ ). This approach was already suggested in [7] and relies on the physical argumentations of Herring (see [27, 28]). In [14], the authors choose a quadratic dependence on curvature for $g$ of the form

$$
g(\theta, k):=g(\theta)+\frac{\varepsilon}{2} k^{2},
$$

with $\varepsilon$ denoting a (small) positive constant (see also [24]). Hence, replacing the surface energy density in (1.2) with (1.6) and taking into account the bulk elastic energy (1.4), the total energy of the system at a time $t$ in $[0, T]$, is

$$
F(h):=\int_{\Omega_{h}} W\left(E\left(u_{h}\right)\right) \mathrm{d} z+\int_{\Gamma_{h}}\left(g(\theta)+\frac{\varepsilon}{2} k^{2}\right) \mathrm{d} \mathcal{H}^{1},
$$

where $u_{h}(\cdot, t)$ is the minimizer of the elastic energy (1.4) in $\Omega_{h(\cdot, t)}$ under suitable boundary and periodicity conditions. The resulting parabolic equations are

$$
V=\left(g_{\theta \theta}+g\right) k-W(E(u))-\varepsilon\left(k_{\sigma \sigma}+\frac{1}{2} k^{3}\right) \text { on } \Gamma_{h}
$$

for the evaporation-condensation case, and

$$
V=\left(-\left(g_{\theta \theta}+g\right) k+W(E(u))+\varepsilon\left(k_{\sigma \sigma}+\frac{1}{2} k^{3}\right)\right)_{\sigma \sigma} \text { on } \Gamma_{h}
$$


for the surface diffusion case. These equations have been already proposed in [19], where (1.9) has been analytically studied. To the best of our knowledge, no analytical results exist in literature for (1.8), unless we restrict ourselves to the case without elasticity, as in $[8,9,13,17,39]$ (see also $[5,6])$.

In this paper, we prove short time existence, uniqueness, and regularity of spatially periodic solutions of (1.8). Precisely, we say that $(h, u)$ is a $b$-periodic configuration in $\Omega_{h}$ if $h(\cdot, t)$ is $b$-periodic in $\mathbb{R}$ and $u(x+b, y, t)=u(x, y, t)+\left(e_{0} b, 0\right)$ for each $(x, y)$ in the subgraph of $h(\cdot, t)$ and any time $t \in[0, T]$. Given an initial $b$-periodic profile $h_{0} \in H_{\text {loc }}^{2}(\mathbb{R} ;(0, \infty))$, we find a time $T_{0}>0$ such that, for each $T<T_{0}$, there exists a unique solution $(h, u)$ of the Cauchy problem

$$
\left\{\begin{array}{l}
\frac{1}{J} h_{t}=\left(g_{\theta \theta}+g\right) k-W(E(u))-\varepsilon\left(k_{\sigma \sigma}+\frac{1}{2} k^{3}\right) \text { in } \mathbb{R} \times(0, T) \\
\operatorname{div} \mathbb{C} E(u)=0 \text { in } \Omega_{h} \\
\mathbb{C} E(u)[\nu]=0 \text { on } \Gamma_{h} \text { and } u(x, 0, t)=\left(e_{0} x, 0\right) \\
(h, u) \text { is a } b \text {-periodic configuration in } \Omega_{h} \\
h(\cdot, 0)=h_{0}
\end{array}\right.
$$

where $W(E(u))$ is defined for each $t \in[0, T]$ as the trace of $W(E(u(\cdot, t)))$ on the graph of $h(\cdot, t)$. See the review article [31, Section 4.2.2] where this problem is proposed to find further references.

Since (1.8) can be regarded as the gradient flow of the total energy functional $F$ with respect to the $L^{2}$-metric, we adopt the minimizing movement method introduced by De Giorgi (see $[3,4]$ ). The idea is based on the discretization of the time interval $[0, T]$ in $N \in \mathbb{N}$ subintervals with length $\tau_{N}$, and on defining inductively the approximate solution $h_{N}$ at time $i \tau_{N}$ by a minimum problem that depends on the approximate solution at the previous time. Precisely, we start with the initial profile $h_{N}(\cdot, 0):=h_{0}$ and for each $i=1, \ldots, N$, we find $h_{N}\left(\cdot, i \tau_{N}\right)$ as the minimizer of

$$
F(h)+\frac{1}{2 \tau_{N}} d^{2}\left(h, h_{N}\left(\cdot,(i-1) \tau_{N}\right)\right)
$$

where the function $d$, that measures the $L^{2}$-distance between $h$ and $h_{N}\left(\cdot,(i-1) \tau_{N}\right)$, is chosen so that the Euler equation of this minimum problem corresponds to a time discretization of (1.8) (see (3.23) below). Then, the discrete-time evolution $h_{N}$ is defined in $[0, T]$ as the piecewise constant or linear interpolant of $\left\{h_{N}\left(\cdot, i \tau_{N}\right)\right\}$. This approach was already adopted in [2] to deal with the motion of crystalline boundaries by mean curvature. Moreover, minimizing movements have been used also more recently to study mean curvature type flows in the case without elasticity in $[8,11,13]$, and for the equation (1.9) in [19] (see also [37] for the Hele-Shaw equation and [16]). As already observed in [12], the basic differences between the evaporation-condensation and the surface diffusion evolution equations are that the latter preserves the area underneath the film profile and it is a gradient flow of $F$ with respect to another metric, the $H^{-1}$-distance (see also [40]). 
The paper is organized as follows. In Section 2 we introduce the incremental minimum problem (1.11) choosing the appropriate function $d$ (see the penalization term (2.7)), and we prove the existence of the discrete-time evolutions. Since in the evaporation-condensation case there are no constraints on the area of $\Omega_{h}$, we proceed in a different way with respect to [19]. In fact, following an argument in [22, Chapter 12], we find $h_{N}$ among functions with spatial derivative uniformly bounded by some constant $r>0$. In particular, we start considering admissible profile functions in $H_{\text {loc }}^{2}(\mathbb{R} ;[0, \infty))$.

In Section 3 we prove that for each $T$ and $r$, the corresponding discrete-time evolutions $h_{N}$ converge to a function $h$ in $C^{0, \beta}\left([0, T] ; C^{1, \alpha}([0, b])\right)$ for every $\alpha \in\left(0, \frac{1}{2}\right)$ and $\beta \in$ $\left(0, \frac{1-2 \alpha}{8}\right)$. Furthermore, since we prove that $\left\{h_{N}\right\}$ is equicontinuous in time with respect to the $C^{1, \alpha}$-metric, we are allowed to select a time $T_{0}$ small enough and $r_{0}$ such that $h_{N}$ is a weak solution of the time discretization of (1.8) for each $T<T_{0}$ (see (3.23)). Then, using the time discretization of (1.8) to estimate higher order derivatives of $h_{N}$, we prove that $h \in L^{2}\left(0, T ; H_{\text {loc }}^{4}(\mathbb{R})\right) \cap H^{1}\left(0, T ; L_{\text {loc }}^{2}(\mathbb{R})\right)$. Finally, in Theorem 3.9 we prove that $h$ is a solution of (1.10), and in Theorem 3.10 we state the regularity properties satisfied by $h$. Furthermore, this method provides an estimate of the $L^{\infty}\left(0, T ; L^{\infty}(\mathbb{R})\right)$-norm of $h_{x}$ in terms of $\left\|h_{0}^{\prime}\right\|_{\infty}$.

This existence result appears to be the first in the presence of elasticity and without surface diffusion. Moreover, we believe that the method is so general that could be applied also to the case with surface diffusion (1.9) to prove a short time existence and regularity result without the use of constant speed parametrizations of the profiles.

Finally, in Section 4 we prove that the solution found with the minimizing movement method is the unique solution of $(1.10)$ in $[0, T]$ with $T<T_{0}$. Since (1.8) does not necessarily preserve the area underneath the profile of the film, the proof is more involved than the one in [19] for the case with surface diffusion.

The study of the long time existence and the global behavior of the solution of (1.8), as well as the asymptotic stability, will be the subject of future work.

\section{Mathematical Setting}

In this section we introduce the precise mathematical formulation of the problem. Following the literature (see $[10,19]$ ), we consider periodic conditions on the evolving profile and on the corresponding elastic displacement. Given a constant $b>0$, we denote by $H_{\#}^{m}(0, b)$, for $m=0,1, \ldots$, the space of all functions in $H_{\mathrm{loc}}^{m}(\mathbb{R})$ that are $b$-periodic, endowed with the norm in $H^{m}(0, b)$. The class of admissible profile functions is

$$
A P:=\left\{h: \mathbb{R} \rightarrow[0, \infty): h \in H_{\#}^{2}(0, b)\right\},
$$

for a positive constant $b$. Furthermore, given $h \in A P$,

$$
\Gamma_{h}:=\{z=(x, h(x)): 0<x<b\} \quad \text { and } \quad \Omega_{h}:=\{z=(x, y): 0<x<b, 0<y<h(x)\}
$$

denote, respectively, the profile and the reference configuration of the film with respect to the interval $(0, b)$, while the corresponding sets on all the domain $\mathbb{R}$ are denoted by $\Gamma_{h}^{\#}$ and 
$\Omega_{h}^{\#}$. Moreover, the class of admissible planar displacements is

$$
\begin{array}{r}
A D_{h}:=\left\{u: \Omega_{h}^{\#} \rightarrow \mathbb{R}^{2}: u \in H^{1}\left(\Omega_{h} ; \mathbb{R}^{2}\right), u(\cdot, 0)=\left(e_{0} \cdot 0\right)\right. \text { in the sense of traces, } \\
\left.\quad \text { and } u(x+b, y)=u(x, y)+\left(e_{0} b, 0\right) \text { for a.e. }(x, y) \in \Omega_{h}^{\#}\right\},
\end{array}
$$

$$
\text { and } \left.u(x+b, y)=u(x, y)+\left(e_{0} b, 0\right) \text { for a.e. }(x, y) \in \Omega_{h}\right\}
$$

where the constant $e_{0}>0$ represents the mismatch between the lattices of the film and the substrate. Consequently, the functional space of admissible configurations is

$$
X_{e_{0}}:=\left\{(h, u): h \in A P, u \in A D_{h}\right\} .
$$

As in [19], we define the surface energy density $g:[0,2 \pi] \rightarrow(0, \infty)$ by

$$
g(\theta):=\psi(\cos \theta, \sin \theta)
$$

where $\psi: \mathbb{R}^{2} \rightarrow(0, \infty)$ is a positively one-homogeneous function of class $C^{2}$ away from the origin. Note that these are the only hypotheses assumed on $\psi$ throughout the paper. From these assumptions it follows that there exists a constant $C>0$ such that

$$
\frac{1}{C}|\xi| \leq \psi(\xi) \leq C|\xi|
$$

for each $\xi \in \mathbb{R}^{2}$.

We recall that $W: \mathbb{M}_{\text {sym }}^{2 \times 2} \rightarrow[0, \infty)$ is defined by

$$
W(A):=\frac{1}{2} \mathbb{C} A: A
$$

with $\mathbb{C}$ a constant positive definite fourth-order tensor, and thus the total energy functional (1.7) becomes

$$
F(h, u):=\int_{\Omega_{h}} W(E(u)) \mathrm{d} z+\int_{\Gamma_{h}}\left(\psi(\nu)+\frac{\varepsilon}{2} k^{2}\right) \mathrm{d} \mathcal{H}^{1},
$$

for each $(h, u) \in X_{e_{0}}$, where $E(u):=\frac{1}{2}\left(\nabla u+\nabla^{T} u\right), \nu$ is the outer normal vector to $\Omega_{h}, k$ is the curvature of $\Gamma_{h}$, and $\varepsilon$ is a (small) positive constant. In particular, given $h \in A P$, we have that

$$
k=\left(\frac{h^{\prime}}{\sqrt{1+\left(h^{\prime}\right)^{2}}}\right)^{\prime} \quad \text { and } \quad \nu=\frac{\left(-h^{\prime}, 1\right)}{\sqrt{1+\left(h^{\prime}\right)^{2}}} .
$$

Consider a non-identically zero profile $h \in A P$ and introduce the elastic energy

$$
\int_{\Omega_{h}} W(E(v)) \mathrm{d} z
$$

defined for each $v \in A D_{h}$. Then there exists a minimizer of (2.5) in $A D_{h}$ (see Lemma 5.1) that is unique due to the Dirichlet condition.

Definition 2.1. Given $h \in A P$ with $h \not \equiv 0$, we say that $u \in A D_{h}$ is the elastic equilibrium corresponding to $h$ if $u$ minimizes (2.5) among all $v \in A D_{h}$. Moreover, $\left(h_{0}, u_{0}\right) \in X_{e_{0}}$ is said to be an initial configuration if $h_{0} \neq \equiv$ and $u_{0}$ is the elastic equilibrium corresponding to $h_{0}$. 
Consider an initial configuration $\left(h_{0}, u_{0}\right) \in X_{e_{0}}$, fix $r>\left\|h_{0}^{\prime}\right\|_{\infty}, T>0, N \in \mathbb{N}$, and set

$$
\tau_{N}:=T / N \text {. }
$$

We now introduce the iterative minimization process used to define the discrete-time evolutions.

The incremental minimum problem. Set $\left(h_{0, N}^{r}, u_{0, N}^{r}\right):=\left(h_{0}, u_{0}\right)$, and for $i=1, \ldots, N$, define inductively $\left(h_{i, N}^{r}, u_{i, N}^{r}\right)$ as a solution of the following minimum problem:

$$
\min \left\{G_{i, N}(h, u):(h, u) \in X_{e_{0}} \text { and }\left\|h^{\prime}\right\|_{\infty} \leq r\right\} .
$$

The functional $G_{i, N}$ is given by

$$
G_{i, N}(h, u):=F(h, u)+P_{i, N}(h),
$$

with the penalization term $P_{i, N}$ defined by

$$
P_{i, N}(h):=\frac{1}{2 \tau_{N}} \int_{\Gamma_{h_{i-1, N}^{r}}}\left(\frac{h-h_{i-1, N}^{r}}{J_{i-1, N}^{r}}\right)^{2} \mathrm{~d} \mathcal{H}^{1}=\frac{1}{2 \tau_{N}} \int_{0}^{b} \frac{\left(h-h_{i-1, N}^{r}\right)^{2}}{J_{i-1, N}^{r}} \mathrm{~d} x,
$$

where $J_{i-1, N}^{r}:=\sqrt{1+\left(\left(h_{i-1, N}^{r}\right)^{\prime}\right)^{2}}$.

The incremental minimum problem is well defined. In fact, for each $i=1, \ldots, N$, we can recursively find a solution of the minimum problem $\left(M_{i, N}^{r}\right)$ as it is established by the following result.

Theorem 2.2. Let $\left(h_{0}, u_{0}\right) \in X_{e_{0}}$ be an initial configuration and let $r>\left\|h_{0}^{\prime}\right\|_{\infty}, T>0$ and $N \in \mathbb{N}$. Then, for $i=1, \ldots, N$, the minimum problem $\left(M_{i, N}^{r}\right)$ admits a solution $\left(h_{i, N}^{r}, u_{i, N}^{r}\right) \in X_{e_{0}}$ with $\left\|\left(h_{i, N}^{r}\right)^{\prime}\right\|_{\infty} \leq r$.

Proof. Fix $i=1, \ldots, N$, and if $i>1$, consider a solution $\left(h_{j, N}^{r}, u_{j, N}^{r}\right)$ of $\left(M_{j, N}^{r}\right)$ for each $j=1, \ldots, i-1$. We want to find a solution of $\left(M_{i, N}^{r}\right)$. First observe that by $(2.6),(2.7)$, and by the minimality of $\left(h_{j, N}^{r}, u_{j, N}^{r}\right)$, we have

$$
F\left(h_{j, N}^{r}, u_{j, N}^{r}\right) \leq G_{j, N}\left(h_{j, N}^{r}, u_{j, N}^{r}\right) \leq G_{j, N}\left(h_{j-1, N}^{r}, u_{j-1, N}^{r}\right)=F\left(h_{j-1, N}^{r}, u_{j-1, N}^{r}\right),
$$

and so

$$
0 \leq \inf _{(h, u) \in X_{e_{0}}} G_{i, N}(h, u) \leq G_{i, N}\left(h_{i-1, N}^{r}, u_{i-1, N}^{r}\right)=F\left(h_{i-1, N}^{r}, u_{i-1, N}^{r}\right) \leq \cdots \leq F\left(h_{0}, u_{0}\right) .
$$

Therefore, we are allowed to select a minimizing sequence $\left\{\left(h_{n}, u_{n}\right)\right\} \subset X_{e_{0}}$ for $\left(M_{i, N}^{r}\right)$ such that $\left\|h_{n}^{\prime}\right\|_{\infty} \leq r$ for each $n$ and $\sup G_{i, N}\left(h_{n}, u_{n}\right)<\infty$.

Since $\sup _{n} P_{i, N}\left(h_{n}, u_{n}\right)<\infty$ and $J_{i-1, N}^{r} \leq \sqrt{1+r^{2}}$, we have that $\left\{h_{n}\right\}$ is bounded in $L^{2}(0, b)$ (by a constant depending on $r$ ). Furthermore, $\left\{h_{n}\right\}$ is bounded in $H^{2}(0, b)$ since 
$\left\|h_{n}^{\prime}\right\|_{\infty} \leq r$ and

$$
\frac{\varepsilon}{2\left(1+r^{2}\right)^{\frac{5}{2}}}\left\|h_{n}^{\prime \prime}\right\|_{L^{2}([0, b])}^{2} \leq \frac{\varepsilon}{2} \int_{0}^{b} \frac{\left(h_{n}^{\prime \prime}\right)^{2}}{\left(1+\left(h_{n}^{\prime}\right)^{2}\right)^{\frac{5}{2}}} \mathrm{~d} x=\frac{\varepsilon}{2} \int_{\Gamma_{h_{n}}} k^{2} \mathrm{~d} \mathcal{H}^{1}<\infty .
$$

Thus, there exists $h \in A P$ with $\left\|h^{\prime}\right\|_{\infty} \leq r$ such that, up to a subsequence (not relabeled), $h_{n} \rightarrow h$ in $H^{2}(0, b)$ and $h_{n} \rightarrow h$ in $W^{1, \infty}(0, b)$. Using Fatou's Lemma, we conclude that

$$
P_{i, N}(h) \leq \liminf _{n \rightarrow \infty} P_{i, N}\left(h_{n}\right),
$$

and in view of the continuity of $\psi$, we have

$$
\int_{\Gamma_{h}} \psi(\nu) \mathrm{d} \mathcal{H}^{1}=\int_{0}^{b} \psi\left(-h^{\prime}, 1\right) \mathrm{d} x \leq \liminf _{n \rightarrow \infty} \int_{0}^{b} \psi\left(-h_{n}^{\prime}, 1\right) \mathrm{d} x=\liminf _{n \rightarrow \infty} \int_{\Gamma_{h_{n}}} \psi(\nu) \mathrm{d} \mathcal{H}^{1},
$$

where in the first and last equality we used the fact that $\psi$ is positively one-homogeneous. Furthermore, since $\left(1+(\cdot)^{2}\right)^{-\frac{5}{4}}$ is uniformly continuous on $[-r, r]$, the sequence $\{(1+$ $\left.\left.\left(h_{n}^{\prime}\right)^{2}\right)^{-\frac{5}{4}}\right\}$ converges uniformly to $\left(1+\left(h^{\prime}\right)^{2}\right)^{-\frac{5}{4}}$, and so

$$
\frac{h_{n}^{\prime \prime}}{\left(1+\left(h_{n}^{\prime}\right)^{2}\right)^{\frac{5}{4}}} \rightarrow \frac{h^{\prime \prime}}{\left(1+\left(h^{\prime}\right)^{2}\right)^{\frac{5}{4}}} \text { in } L^{2}(0, b)
$$

due to the weak convergence of $\left\{h_{n}^{\prime \prime}\right\}$ in $L^{2}(0, b)$. Thus, we have

$$
\begin{aligned}
\int_{\Gamma_{h}} k^{2} \mathrm{~d} \mathcal{H}^{1} & =\int_{0}^{b} \frac{\left(h^{\prime \prime}\right)^{2}}{\left(1+\left(h^{\prime}\right)^{2}\right)^{\frac{5}{2}}} \mathrm{~d} x \\
& \leq \liminf _{n \rightarrow \infty} \int_{0}^{b} \frac{\left(h_{n}^{\prime \prime}\right)^{2}}{\left(1+\left(h_{n}^{\prime}\right)^{2}\right)^{\frac{5}{2}}} \mathrm{~d} x=\liminf _{n \rightarrow \infty} \int_{\Gamma_{h_{n}}} k^{2} \mathrm{~d} \mathcal{H}^{1}
\end{aligned}
$$

In order to prove that the sequence $\left\{u_{n}\right\}$ is bounded in an appropriate space, we need to apply Lemma 5.1 in the Appendix. For this purpose, we consider a constant

$$
L \geq \sup _{n}\left\|h_{n}\right\|_{C^{1}([0, b])},
$$

we define a set $U:=(0, b) \times(0,-L(1+3 b))$, and we choose $w \in H^{1}\left(U ; \mathbb{R}^{2}\right)$ with null trace on $(0, b) \times\{-L(1+3 b)\}$ and trace equal to $\left(e_{0}, 0\right)$ on $(0, b) \times\{0\}$ such that

$$
\|w\|_{H^{1}\left(U ; \mathbb{R}^{2}\right)} \leq C\|\operatorname{Tr}(w)\|_{H^{\frac{1}{2}}(\partial U)}
$$

for some constant $C>0$ (see [29]), where $\operatorname{Tr}(\cdot)$ is the trace operator. We may now extend each $u_{n}$ to $U_{h_{n}}:=\left\{z=(x, y): 0<x<b,-L(1+3 b)<y<h_{n}(x)\right\}$ with $w$, without relabeling it. Applying Lemma 5.1 to each $U_{h_{n}}$, we obtain

$$
\int_{U_{h_{n}}}\left|u_{n}\right|^{2} \mathrm{~d} z+\int_{U_{h_{n}}}\left|\nabla u_{n}\right|^{2} \mathrm{~d} z \leq C\left(\int_{\Omega_{h_{n}}}\left|E\left(u_{n}\right)\right|^{2} \mathrm{~d} z+\|\operatorname{Tr}(w)\|_{H^{\frac{1}{2}}(\partial U)}^{2}\right)
$$


for some constant $C>0$ depending only on $L$. Therefore, since $\sup _{n} \int_{\Omega_{h_{n}}}\left|E\left(u_{n}\right)\right|^{2} \mathrm{~d} z<\infty$, we have that $\left\|u_{n}\right\|_{H^{1}\left(U_{h_{n}} ; \mathbb{R}^{2}\right)}$ are equibounded. Proceeding now as in Lemma 5.1, since each $U_{h_{n}}$ has Lipschitz boundary, we extend $u_{n}$ to the rectangle $R_{L}:=(0, b) \times(-L(1+$ $3 b), L(1+3 b)$ ) and we obtain that, up to a subsequence (not relabeled), $\left\{u_{n}\right\}$ converges weakly in $H^{1}\left(R_{L} ; \mathbb{R}^{2}\right)$ to some function $u$ with trace equal to $\left(e_{0} \cdot, 0\right)$ on $(0, b) \times\{0\}$ (see [29]). Furthermore, we extend $u$ to $\Omega_{h}^{\#}$ by defining $u(x+b, y):=u(x, y)+\left(e_{0} b, 0\right)$ for every $(x, y) \in \Omega_{h}^{\#} \backslash \Omega_{h}$, so that $(h, u) \in X_{e_{0}}$.

Finally, since $\left\{E\left(u_{n}\right)\right\}$ weakly converges to $E(u)$ in $L^{2}\left(R_{L} ; \mathbb{R}^{2}\right)$ and $\left\{h_{n}\right\}$ convergences uniformly to $h$, we conclude that

$$
\int_{\Omega_{h}} W(E(u)) \mathrm{d} z \leq \liminf _{n \rightarrow \infty} \int_{\Omega_{h_{n}}} W\left(E\left(u_{n}\right)\right) \mathrm{d} z
$$

which, together with $(2.9),(2.10)$ and $(2.11)$, implies that $(h, u)$ is a minimizer of $\left(M_{i, N}^{r}\right)$.

Remark 2.3. Let $f \in H^{\frac{1}{2}}(0, b)$. The previous theorem still holds true if we replace the Dirichlet boundary condition $u(\cdot, 0)=\left(e_{0} \cdot, 0\right)$ in $(2.1)$ with the more general condition $u(\cdot, 0)=(f(\cdot), 0)$. Precisely, let $h_{0} \in H^{2}(0, b)$ be an initial profile and let $r>\left\|h_{0}^{\prime}\right\|_{\infty}, T>0$ and $N \in \mathbb{N}$. Then, for $i=1, \ldots, N$, the functional (2.6) admits a minimizer in

$$
X_{f}^{r}:=\left\{(u, h): h \in H^{2}(0, b) \text { with }\left\|h^{\prime}\right\|_{\infty} \leq r, u \in H^{1}\left(\Omega_{h} ; \mathbb{R}^{2}\right) \text { with } u(\cdot, 0)=(f(\cdot), 0)\right\} .
$$

In fact, this result follows from the same arguments used in the previous proof with the only difference that we need now to select the function $w \in H^{1}\left(U ; \mathbb{R}^{2}\right)$ in $(2.12)$ with null trace on $(0, b) \times\{-L(1+3 b)\}$ and trace equal to $(f(\cdot), 0)$ on $(0, b) \times\{0\}$. We choose such a function $w$ by extending $f$ to $\mathbb{R}$ by [15, Theorem 5.4], using the surjectivity of the trace operator from $H^{1}\left(\mathbb{R}_{-}^{2}\right)$ to $H^{\frac{1}{2}}(\mathbb{R})$ (see $[29]$ ), and finally truncating near $\mathbb{R} \times\{-L(1+3 b)\}$ with a cut-off function.

In view of Theorem 2.2 we may define the notion of discrete-time evolution of (1.8).

Definition 2.4. Let $\left(h_{0}, u_{0}\right) \in X_{e_{0}}$ be an initial configuration and let $r>\left\|h_{0}^{\prime}\right\|_{\infty}, T>0$ and $N \in \mathbb{N}$. For $i=1, \ldots, N$, consider a solution $h_{i, N}^{r}$ to $\left(M_{i, N}^{r}\right)$ given by Theorem 2.2. The piecewise linear interpolation $h_{N}^{r}: \mathbb{R} \times[0, T] \rightarrow[0, \infty)$ of the functions $h_{i, N}^{r}$, namely the function defined by

$$
h_{N}^{r}(x, t):=h_{i-1, N}^{r}(x)+\frac{1}{\tau_{N}}\left(t-(i-1) \tau_{N}\right)\left(h_{i, N}^{r}(x)-h_{i-1, N}^{r}(x)\right)
$$

if $(x, t) \in \mathbb{R} \times\left[(i-1) \tau_{N}, i \tau_{N}\right]$, for $i=1, \ldots, N$, is said to be a discrete-time evolution of (1.8). In addition, for each $t \in[0, T]$ we denote by $u_{N}^{r}(\cdot, t)$ the elastic equilibrium corresponding to $h_{N}^{r}(\cdot, t)$.

We observe that, by Theorem 2.2 , if $\left(h_{0}, u_{0}\right) \in X_{e_{0}}$ is an initial configuration, $r>\left\|h_{0}^{\prime}\right\|_{\infty}$ and $T>0$, then for each $N \in \mathbb{N}$ there exists a discrete-time evolution $h_{N}^{r}$ of (1.8) and we have that $h_{N}^{r}(\cdot, t) \in A P$ and $\left\|\frac{\partial h_{N}^{r}}{\partial x}(\cdot, t)\right\|_{\infty} \leq r$ for all $t$ in $[0, T]$. 
Remark 2.5. In what follows, given a regular height function $h: \mathbb{R} \times[0, T] \rightarrow[0, \infty)$, $h_{x}$ and $h_{t}$ stand for the derivatives with respect to the space and the time, respectively. Moreover, for each $t \in[0, T]$, given a regular function $u(\cdot, t): \Omega_{h(\cdot, t)}^{\#} \rightarrow \mathbb{R}^{2}$, we denote by $\nabla u(\cdot, t)$ the gradient of $u$ with respect to the spatial coordinates and by $E(u)(\cdot, t):=$ $\frac{1}{2}\left(\nabla u(\cdot, t)+\nabla^{T} u(\cdot, t)\right)$ its symmetric part. Furthermore, $E(u)(\cdot, h(\cdot, t)): \mathbb{R} \rightarrow \mathbb{M}_{\text {sym }}^{2 \times 2}$ is the trace of $E(u)(\cdot, t)$ on $\Gamma_{h(\cdot, t)}^{\#}$.

We now introduce the notion of a solution of (1.10) in the interval of time $[0, T]$.

Definition 2.6. Let $\left(h_{0}, u_{0}\right) \in X_{e_{0}}$ be an initial configuration. A solution of (1.10) in $[0, T]$ with initial configuration $\left(h_{0}, u_{0}\right)$ is a function $h \in L^{2}\left(0, T ; H_{\#}^{4}(0, b)\right) \cap H^{1}\left(0, T ; L_{\#}^{2}(0, b)\right)$ that satisfies $h(\cdot, 0)=h_{0}(\cdot)$ in $[0, b]$, and

$$
\frac{1}{J} h_{t}=-\varepsilon\left(\frac{h_{x x}}{J^{5}}\right)_{x x}-\frac{5 \varepsilon}{2}\left(\frac{h_{x x}^{2}}{J^{7}} h_{x}\right)_{x}+\partial_{11} \psi\left(-h_{x}, 1\right) h_{x x}-W
$$

in $(0, b) \times(0, T]$, where $J:=\sqrt{1+\left|h_{x}\right|^{2}}, \partial_{11} \psi$ denotes the second derivative of $\psi$ with respect to the first component, $W(\cdot, t):=W(E(u)(\cdot, h(\cdot, t)))$ and $u(\cdot, t)$ is the elastic equilibrium corresponding to $h(\cdot, t)$ for each $t \in[0, T]$.

Note that (2.15) is (1.8) using the parametrization with the height function. Indeed, in this case the curvature, the normal velocity of the evolving profile $\Gamma_{h}$, and the outward normal vector $\nu$ to $\Omega_{h}$ at the point $(\cdot, h(\cdot))$ are given, respectively, by

$$
k=\left(\frac{h_{x}}{\sqrt{1+\left|h_{x}\right|^{2}}}\right)_{x}, \quad V=\frac{1}{J} h_{t} \quad \text { and } \quad \nu=\frac{1}{J}\left(-h_{x}, 1\right),
$$

and $(\cdot)_{\sigma}=\frac{1}{J}(\cdot)_{x}$ (see Lemmas 5.2 and 5.3 in the Appendix).

\section{EXISTENCE AND REGULARITY}

In this section we establish the existence of a solution of (1.10) in the sense of the Definition 2.6 for short time intervals and we study its regularity (see Theorems 3.9 and 3.10). First, we consider an initial configuration $\left(h_{0}, u_{0}\right) \in X_{e_{0}}$ and we prove that, if $\left\{h_{N}^{r}\right\}$ is a sequence of discrete-time evolutions for $r>\left\|h_{0}^{\prime}\right\|_{\infty}$ and $T>0$ (see Definition 2.4), then, up to a subsequence (not relabeled), it converges to some function $h^{r}$ as $N \rightarrow \infty$. Next, we select a time $T_{0}$ small enough and $r_{0}$ appropriate to have that $\left\|\left(h_{i, N}^{r_{0}}\right)^{\prime}\right\|_{\infty}<r_{0}$ for each $T<T_{0}, N \in \mathbb{N}$, and $i=1, \ldots, N$. For $T<T_{0}$ the profile function $h_{i, N}^{r_{0}}$ satisfies the Euler-Lagrange equation (3.23) corresponding to the minimum problem $\left(M_{i, N}^{r_{0}}\right)$. Finally, using the estimates provided by (3.23), we prove that $h^{r_{0}}$ is a solution of $(1.10)$ on $[0, T]$ for $T<T_{0}$.

We begin by showing that the discrete-time evolutions $h_{N}^{r}$ introduced in Definition 2.4 are uniformly bounded in $L^{\infty}\left(0, T ; H^{2}(0, b)\right) \cap H^{1}\left(0, T ; L^{2}(0, b)\right)$. In the following, we pay attention to the dependence on $r$ of the constants involved in the estimates used to select $T_{0}$ in Corollary 3.3. 
Theorem 3.1. Let $\left(h_{0}, u_{0}\right) \in X_{e_{0}}$ be an initial configuration and let $r>\left\|h_{0}^{\prime}\right\|_{\infty}, T>0$ and $N \in \mathbb{N}$. For $i=1, \ldots, N$, consider a solution $h_{i, N}^{r}$ to $\left(M_{i, N}^{r}\right)$ given by Theorem 2.2 and the related discrete-time evolution introduced in Definition 2.4. Then,

$$
\int_{0}^{T} \int_{0}^{b}\left|\frac{\partial h_{N}^{r}}{\partial t}(\cdot, t)\right|^{2} \mathrm{~d} x \mathrm{~d} t \leq C_{0}(r) \quad \text { and } \quad \sup _{i}\left\|h_{i, N}^{r}\right\|_{H^{2}(0, b)} \leq \sqrt{C_{0}(r) T}+C_{1}(r),
$$

where $C_{0}(r), C_{1}(r)>0$ are constants that depend only on $r$.

Therefore, up to a subsequence,

$$
h_{N}^{r} \rightarrow h^{r} \text { in } L^{2}\left(0, T ; H^{2}(0, b)\right) \quad \text { and } \quad h_{N}^{r} \rightarrow h^{r} \text { in } H^{1}\left(0, T ; L^{2}(0, b)\right)
$$

as $N \rightarrow \infty$, for some function $h^{r} \in L^{2}\left(0, T ; H^{2}(0, b)\right) \cap H^{1}\left(0, T ; L^{2}(0, b)\right)$. Moreover, for every $\gamma \in\left(0, \frac{1}{2}\right)$ we have

$$
h_{N}^{r} \rightarrow h^{r} \text { in } C^{0, \gamma}\left([0, T] ; L^{2}(0, b)\right) \text { as } N \rightarrow \infty,
$$

$h^{r} \in L^{\infty}\left(0, T ; H^{2}(0, b)\right), h^{r}(\cdot, t) \in A P$, and $\left\|\frac{\partial h^{r}}{\partial x}(\cdot, t)\right\|_{\infty} \leq r$ for every $t$ in $[0, T]$.

Proof. Fix $r>\left\|h_{0}^{\prime}\right\|_{\infty}, T>0$ and $N \in \mathbb{N}$. For simplicity, in this proof, we disregard the dependence on $r$ in the notation of $h_{i, N}^{r}$ and $h_{N}^{r}$. For each $i=1, \ldots, N$, we have that

$$
G_{i, N}\left(h_{i, N}, u_{i, N}\right) \leq G_{i, N}\left(h_{i-1, N}, u_{i-1, N}\right)=F\left(h_{i-1, N}, u_{i-1, N}\right)
$$

by $(2.6),(2.7)$ and the minimality of $\left(h_{i, N}, u_{i, N}\right)$. Thus, $P_{i, N}\left(h_{i, N}\right) \leq F\left(h_{i-1, N}, u_{i-1, N}\right)-$ $F\left(h_{i, N}, u_{i, N}\right)$ and so,

$$
\frac{1}{2 \tau_{N} \sqrt{1+r^{2}}} \int_{0}^{b}\left(h_{i, N}-h_{i-1, N}\right)^{2} \mathrm{~d} x \leq F\left(h_{i-1, N}, u_{i-1, N}\right)-F\left(h_{i, N}, u_{i, N}\right) .
$$

Recalling (2.14) and summing over $i=1, \ldots, N$, since $F \geq 0$ we obtain

$$
\frac{1}{2 \sqrt{1+r^{2}}} \int_{0}^{T} \int_{0}^{b}\left|\frac{\partial h_{N}}{\partial t}(x, t)\right|^{2} \mathrm{~d} x \mathrm{~d} t \leq F\left(h_{0}, u_{0}\right)
$$

i.e. the first estimate in (3.1) with $C_{0}(r):=2 \sqrt{1+r^{2}} F\left(h_{0}, u_{0}\right)$. Now, since $h_{N}(x, \cdot)$ is absolutely continuous on $[0, T]$, for all $t_{1}, t_{2} \in[0, T]$, with $t_{1}<t_{2}$, using Hölder's inequality and Fubini's Theorem, we have

$$
\begin{aligned}
\left\|h_{N}\left(\cdot, t_{2}\right)-h_{N}\left(\cdot, t_{1}\right)\right\|_{L^{2}(0, b)} & \leq\left(\int_{0}^{b}\left(\int_{t_{1}}^{t_{2}} \frac{\partial h_{N}}{\partial t}(x, t) \mathrm{d} t\right)^{2} \mathrm{~d} x\right)^{\frac{1}{2}} \\
& \leq\left(\int_{t_{1}}^{t_{2}}\left\|\frac{\partial h_{N}}{\partial t}(\cdot, t)\right\|_{L^{2}(0, b)}^{2} \mathrm{~d} t\right)^{\frac{1}{2}}\left(t_{2}-t_{1}\right)^{\frac{1}{2}} .
\end{aligned}
$$

Therefore, from the first estimate in (3.1) we obtain

$$
\left\|h_{N}\left(\cdot, t_{2}\right)-h_{N}\left(\cdot, t_{1}\right)\right\|_{L^{2}(0, b)} \leq \sqrt{C_{0}(r)}\left(t_{2}-t_{1}\right)^{\frac{1}{2}}
$$


and, in particular, selecting $t_{1}=0$ and $t_{2}=i \tau_{N}$, since $h_{N}(\cdot, 0)=h_{0}(\cdot)$ and $h_{N}\left(\cdot, i \tau_{N}\right)=$ $h_{i, N}(\cdot),(3.5)$ implies that $\left\|h_{i, N}\right\|_{L^{2}(0, b)} \leq \sqrt{C_{0}(r)} \sqrt{T}+\left\|h_{0}\right\|_{L^{2}([0, b])}$. Furthermore, from (3.4) we observe that $F\left(h_{i, N}, u_{i, N}\right) \leq F\left(h_{i-1, N}, u_{i-1, N}\right)$ for each $i=1, \ldots, N$, and so,

$$
\frac{\varepsilon}{2\left(1+r^{2}\right)^{\frac{5}{2}}}\left\|\left(h_{i, N}\right)^{\prime \prime}\right\|_{L^{2}([0, b])}^{2} \leq \frac{\varepsilon}{2} \int_{\Gamma_{h_{i, N}^{r}}} k^{2} \mathrm{~d} \mathcal{H}^{1} \leq F\left(h_{i, N}, u_{i, N}\right) \leq \cdots \leq F\left(h_{0}, u_{0}\right) .
$$

where we have used the fact that $\left\|h_{i, N}^{\prime}\right\|_{\infty} \leq r$. Thus,

$$
\left\|h_{i, N}^{\prime \prime}\right\|_{L^{2}(0, b)} \leq C_{2}(r)
$$

for $C_{2}(r):=\sqrt{\frac{2}{\varepsilon} F\left(h_{0}, u_{0}\right)}\left(1+r^{2}\right)^{\frac{5}{4}}$, and the second estimate in (3.1) follows.

Therefore, since

$$
\sup _{t \in[0, T]}\left\|h_{N}(\cdot, t)\right\|_{H^{2}(0, b)} \leq \sqrt{C_{0}(r) T}+C_{1}(r),
$$

up to a subsequence (not relabeled), $h_{N} \rightarrow h$ in $L^{2}\left(0, T ; H^{2}(0, b)\right)$ for some function $h$. On the other hand, the first estimate in (3.1) implies that, up to a further subsequence (not relabeled), $\left\{\frac{\partial h_{N}}{\partial t}\right\}$ converges weakly in $L^{2}\left(0, T ; L^{2}(0, b)\right)$, and we deduce that $\frac{\partial h}{\partial t} \in$ $L^{2}\left(0, T ; L^{2}(0, b)\right)$, i.e., $h \in H^{1}\left(0, T ; L^{2}(0, b)\right)$. Finally, note that $(3.5)$ togheter with AscoliArzelà Theorem (see e.g. [4, Proposition 3.3.1]), implies (3.3). Thus, since by (3.7) for each $t$ in $[0, T]$, we can find a sequence $\left\{h_{N_{k}}(\cdot, t)\right\}$ that converges in $W^{1, \infty}(0, b)$, by the uniqueness of the limit we have that $h(\cdot, t) \in A P$ and $\left\|\frac{\partial h}{\partial x}(\cdot, t)\right\|_{\infty} \leq r$.

From now on, we denote by $\left\{h_{N}^{r}\right\}$ and $h^{r}$, respectively, a subsequence and a limit function provided by Theorem 3.1. In the next result we improve the convergence of $\left\{h_{N}^{r}\right\}$ to $h^{r}$.

Theorem 3.2. Let $\left(h_{0}, u_{0}\right) \in X_{e_{0}}$ be an initial configuration. For $r>\left\|h_{0}^{\prime}\right\|_{\infty}, T>0$, we have that $h^{r} \in C^{0, \beta}\left([0, T] ; C^{1, \alpha}([0, b])\right)$ and

$$
h_{N}^{r} \rightarrow h^{r} \text { in } C^{0, \beta}\left([0, T] ; C^{1, \alpha}([0, b])\right) \text { as } N \rightarrow \infty
$$

for every $\alpha \in\left(0, \frac{1}{2}\right)$ and $\beta \in\left(0, \frac{1-2 \alpha}{8}\right)$. Furthermore, $h^{r}(\cdot, t) \rightarrow h_{0}$ in $C^{1, \alpha}([0, b])$ as $t \rightarrow 0^{+}$.

Proof. Fix $r>\left\|h_{0}^{\prime}\right\|_{\infty}, T>0$ and $N \in \mathbb{N}$. In this proof, we disregard again the dependence on $r$ in the notation of $h_{i, N}^{r}$ and $h_{N}^{r}$. Since for each $t_{1}, t_{2}$ in [0,T], with $t_{1}<t_{2}$, the function $g:=h_{N}\left(\cdot, t_{2}\right)-h_{N}\left(\cdot, t_{1}\right)$ is $b$-periodic, by the interpolation inequality (5.8), we have that

$$
\left\|g^{\prime}\right\|_{\infty} \leq K\left\|g^{\prime \prime}\right\|_{L^{2}(0, b)}^{\frac{3}{4}}\|g\|_{L^{2}(0, b)}^{\frac{1}{4}}
$$

for some constant $K>0$, and since $\left\|g^{\prime \prime}\right\|_{L^{2}(0, b)} \leq 2 \sup _{i, N}\left\|h_{i, N}^{\prime \prime}\right\|_{L^{2}(0, b)}$, we obtain

$$
\left\|g^{\prime}\right\|_{\infty} \leq K\left(2 C_{2}(r)\right)^{\frac{3}{4}}\|g\|_{L^{2}(0, b)}^{\frac{1}{4}}
$$


where we used (3.6). Thus, by (3.5) we find that

$$
\left\|\frac{\partial h_{N}}{\partial x}\left(\cdot, t_{2}\right)-\frac{\partial h_{N}}{\partial x}\left(\cdot, t_{1}\right)\right\|_{\infty} \leq C_{3}(r)\left(t_{2}-t_{1}\right)^{\frac{1}{8}},
$$

for $C_{3}(r):=2^{\frac{3}{4}} K C_{2}^{\frac{3}{4}}(r) C_{0}^{\frac{1}{8}}(r)>0$.

Furthermore, by the Mean Value Theorem there exists $\bar{x} \in[0, b]$ such that

$$
g(\bar{x})=\frac{1}{b} \int_{0}^{b} g(x) \mathrm{d} x,
$$

and so

$$
|g(x)| \leq|g(x)-g(\bar{x})|+|g(\bar{x})| \leq b\left\|g^{\prime}\right\|_{\infty}+\frac{1}{\sqrt{b}}\|g\|_{L^{2}(0, b)},
$$

for each $x \in[0, b]$. Therefore, by (3.5) and (3.10), we obtain

$$
\left\|h_{N}\left(\cdot, t_{2}\right)-h_{N}\left(\cdot, t_{1}\right)\right\|_{\infty} \leq C_{3}(r) b\left(t_{2}-t_{1}\right)^{\frac{1}{8}}+\sqrt{\frac{C_{0}(r)}{b}}\left(t_{2}-t_{1}\right)^{\frac{1}{2}} .
$$

Moreover, for every $\alpha \in\left(0, \frac{1}{2}\right)$, if $|\cdot|_{\alpha}$ denotes the $\alpha$-Hölder seminorm, we have

$$
\left|g^{\prime}\right|_{\alpha}:=\sup \left\{\frac{\left|g^{\prime}(x)-g^{\prime}(y)\right|}{|x-y|^{\alpha}}: x, y \in[0, b], x \neq y\right\} \leq\left|g^{\prime}\right|_{\frac{1}{2}}^{2 \alpha}\left(2\left\|g^{\prime}\right\|_{\infty}\right)^{1-2 \alpha} .
$$

Since (3.7) implies that

$$
\left|\frac{\partial h_{N}}{\partial x}\left(\cdot, t_{2}\right)-\frac{\partial h_{N}}{\partial x}\left(\cdot, t_{1}\right)\right|_{\frac{1}{2}} \leq 2 K_{M}\left(\sqrt{C_{0}(r) T}+C_{1}(r)\right)
$$

where $K_{M}$ is the constant of the Morrey's inequality (see [1, 29]), by (3.10) and (3.12) we deduce that

$$
\left|\frac{\partial h_{N}}{\partial x}\left(\cdot, t_{2}\right)-\frac{\partial h_{N}}{\partial x}\left(\cdot, t_{1}\right)\right|_{\alpha} \leq C_{4}(r, \alpha, T)\left(t_{2}-t_{1}\right)^{\frac{1-2 \alpha}{8}},
$$

for $C_{4}(r, \alpha, T):=2 K_{M}^{2 \alpha}\left(\sqrt{C_{0}(r) T}+C_{1}(r)\right)^{2 \alpha}\left(C_{3}(r)\right)^{1-2 \alpha}>0$.

Therefore, it follows from (3.10), (3.11), and (3.13), that for every $\alpha \in\left(0, \frac{1}{2}\right), h_{N}$ is uniformly equicontinuous with respect to the $C^{1, \alpha}([0, b])$-norm topology and that

$$
\left\|h_{N}\left(\cdot, t_{2}\right)-h_{N}\left(\cdot, t_{1}\right)\right\|_{C^{1, \alpha}([0, b])} \leq C(r, \alpha, T)\left(t_{2}-t_{1}\right)^{\frac{1-2 \alpha}{8}},
$$

for some $C(r, \alpha, T)>0$. In particular, we find (3.8) applying Ascoli-Arzelà Theorem (see e.g. $\left[4\right.$, Proposition 3.3.1]). Finally, since $\left\|h_{N}(\cdot, t)-h_{N}\left(\cdot, t_{1}\right)\right\|_{C^{1, \alpha}([0, b])} \rightarrow 0$ as $t \rightarrow t_{1}$, we conclude the proof choosing $t_{1}=0$.

It follows from the previous theorem, that we can select $r_{0}$ and a small time $T_{1}$ (the largest one with respect to the estimate (3.10)) so that $\left\|\frac{\partial h_{N}^{r_{0}}}{\partial x}\right\|_{L^{\infty}([0, b] \times[0, T])}<r_{0}$ for every $T<T_{1}$ and $N \in \mathbb{N}$. 
Corollary 3.3. Let $\left(h_{0}, u_{0}\right) \in X_{e_{0}}$ be an initial configuration, and set

$$
r_{0}:=\left\|h_{0}^{\prime}\right\|_{\infty}+\sqrt{\left\|h_{0}^{\prime}\right\|_{\infty}^{2}+1} \quad \text { and } \quad T_{1}:=\frac{\left(1+\left\|h_{0}^{\prime}\right\|_{\infty}^{2}\right)^{4}}{\sigma_{0}(\varepsilon)\left(1+r_{0}^{2}\right)^{8}},
$$

where $\sigma_{0}(\varepsilon):=2^{10} K^{8} \varepsilon^{-3} F^{4}\left(h_{0}, u_{0}\right)$ and $K$ is the interpolation constant in (3.9). Then, for $T<T_{1}$ we have that $\sup _{i, N}\left\|\left(h_{i, N}^{r_{0}}\right)^{\prime}\right\|_{\infty}<r_{0}$.

Proof. We recall that the constant in $(3.10)$ is $C_{3}(r):=K\left(2 C_{2}(r)\right)^{\frac{3}{4}} C_{0}^{\frac{1}{8}}(r)$, where $K$ is the interpolation constant in $(3.9), C_{0}(r):=2 \sqrt{1+r^{2}} F\left(h_{0}, u_{0}\right)$ and $C_{2}(r):=\sqrt{\frac{2}{\varepsilon} F\left(h_{0}, u_{0}\right)}(1+$ $\left.r^{2}\right)^{\frac{5}{4}}$. Hence, $C_{3}(r)=\sigma_{0}^{\frac{1}{8}}(\varepsilon)\left(1+r^{2}\right)$. Therefore, choosing $t_{1}=0$ and $t_{2}=i \tau_{N}$ in (3.10) we find that

$$
\left\|\left(h_{i, N}^{r}\right)^{\prime}\right\|_{\infty} \leq\left(1+r^{2}\right)\left(\sigma_{0}(\varepsilon) T\right)^{\frac{1}{8}}+\left\|h_{0}^{\prime}\right\|_{\infty},
$$

for $N \in \mathbb{N}$ and $i=1, \ldots, N$. Thus, if $r>\left\|h_{0}^{\prime}\right\|_{\infty}$ then it follows that $\sup _{i, N}\left\|\left(h_{i, N}^{r}\right)^{\prime}\right\|_{\infty}<r$ for every $T<T_{1}(r)$, where

$$
T_{1}(r):=\frac{\left(r-\left\|h_{0}^{\prime}\right\|_{\infty}\right)^{8}}{\sigma_{0}(\varepsilon)\left(1+r^{2}\right)^{8}} .
$$

Choose $r_{0}:=\left\|h_{0}^{\prime}\right\|_{\infty}+\sqrt{\left\|h_{0}^{\prime}\right\|_{\infty}^{2}+1}$ to maximize $T_{1}(r)$ and let $T_{1}:=T_{1}\left(r_{0}\right)$.

Remark 3.4. If $h_{0}>0$ then there exists a time $T_{2}=T_{2}\left(h_{0}\right)>0$ such that $h_{N}^{r_{0}}>0$ in $[0, b] \times[0, T]$ for every $T<T_{2}$. Indeed, by (3.11) with $t_{1}=0$ and $t_{2}=t$ we have that

$$
h_{N}^{r_{0}}(x, t) \geq h_{0}(x)-C_{3}\left(r_{0}\right) b t^{\frac{1}{8}}-\sqrt{\frac{C_{0}\left(r_{0}\right)}{b}} t^{\frac{1}{2}} \geq \min _{x \in[0, b]} h_{0}(x)-C_{3}\left(r_{0}\right) b T^{\frac{1}{8}}-\sqrt{\frac{C_{0}\left(r_{0}\right)}{b}} T^{\frac{1}{2}}
$$

for every $(x, t) \in[0, b] \times[0, T]$.

Define

$$
T_{0}:=\min \left\{T_{1}, T_{2}\right\},
$$

and note that Theorems 3.1 and 3.2 hold true for $r_{0}$ and every $T<T_{0}$. In the rest of the paper we assume that $T<T_{0}$ and, to simplify the notation, we denote $h:=h^{r_{0}}, h_{N}:=h_{N}^{r_{0}}$, $h_{i, N}:=h_{i, N}^{r_{0}}, J_{i, N}^{r_{0}}:=J_{i, N}, u_{N}:=u_{N}^{r_{0}}$ and $u_{i, N}:=u_{i, N}^{r_{0}}$ for all $N \in \mathbb{N}$ and $i=1, \ldots, N$.

Moreover, for technical reasons, in the sequel we use the piecewise constant interpolations of $\left\{J_{i, N}\right\}$, and $\left\{V_{i, N}\right\}$, where $V_{i, N}$ is defined by

$$
V_{i, N}(x):=\frac{1}{\tau_{N}} \frac{h_{i, N}(x)-h_{i-1, N}(x)}{J_{i-1, N}(x)}
$$

for every $x \in \mathbb{R}, i=1, \ldots, N$ and $N \in \mathbb{N}$. We will also use the piecewise constant interpolations for $\left\{u_{i, N}\right\}$ and $\left\{h_{i, N}\right\}$, in place of the piecewise linear interpolations introduced in $(2.14)$. 
Definition 3.5. Let $\left(h_{0}, u_{0}\right) \in X_{e_{0}}$ be an initial configuration, and for $N \in \mathbb{N}$ and $i=$ $1, \ldots, N$, consider $I_{i, N}:=\left((i-1) \tau_{N}, i \tau_{N}\right]$. Define $\tilde{u}_{N}(z, 0):=u_{0}$ for all $z \in \Omega_{h_{0}}$ and

$$
\tilde{u}_{N}(z, t):=u_{i, N}(z) \quad \text { for all } \quad z \in \Omega_{h_{i, N}} \quad \text { if } t \in I_{i, N} \text {. }
$$

Analogously, define $\tilde{h}_{N}$ and $V_{N}: \mathbb{R} \times(0, T] \rightarrow[0, \infty)$ by, respectively,

$$
\tilde{h}_{N}(\cdot, t):=h_{i, N} \quad \text { and } \quad V_{N}(\cdot, t):=V_{i, N} \quad \text { if } \quad t \in I_{i, N} .
$$

In addition, set $\tilde{J}_{N}:=\sqrt{1+\left(\frac{\partial \tilde{h}_{N}}{\partial x}\right)^{2}}$.

Remark 3.6. Fix $T<T_{0}$. In view of Theorem 3.2, we deduce the following convergence results for $\left\{\tilde{h}_{N}\right\},\left\{\tilde{J}_{N}\right\}$ and $\left\{V_{N}\right\}$.

(i) For $\alpha \in\left(0, \frac{1}{2}\right)$,

$$
\tilde{h}_{N} \rightarrow h \text { in } L^{\infty}\left(0, T ; C^{1, \alpha}([0, b])\right),
$$

as $N \rightarrow \infty$. This can be easily verified using the equicontinuity of the sequence $\left\{h_{N}\right\}$ with respect to the $C^{1, \alpha}([0, b])$-norm topology (see $(3.14)$ ).

(ii) It follows from (i) that $\tilde{J}_{N} \rightarrow J:=\sqrt{1+\left|h_{x}\right|^{2}}$ in $L^{\infty}(0, T ; C([0, b]))$.

(iii) Furthermore,

$$
V_{N} \rightarrow V:=\frac{1}{J} h_{t} \text { in } L^{2}\left(0, T ; L^{2}(0, b)\right) .
$$

Indeed, from Definition 2.4 we have that for all $t \in\left((i-1) \tau_{N}, i \tau_{N}\right), x \in \mathbb{R}$,

$$
V_{N}(x, t)=\frac{1}{J_{i-1, N}(x)} \frac{\partial h_{N}}{\partial t}(x, t) .
$$

Hence, (3.20) follows from (ii) and the fact that $\frac{\partial h_{N}}{\partial t} \rightarrow \frac{\partial h}{\partial t}$ in $L^{2}\left(0, T ; L^{2}(0, b)\right)$ by the second assertion in (3.2).

For the convergence of $\left\{u_{N}\right\}$ and $\left\{\tilde{u}_{N}\right\}$, we follow the last part of the proof of $[19$, Theorem 3.4], where standard elliptic estimates are used (see [21, Proposition 8.9]). In the remainder of the paper, we assume that the initial profile is strictly positive, i.e.,

$$
h_{0}>0 \text {, }
$$

and we use the notation introduced in Remark 2.5.

Theorem 3.7. Let $\left(h_{0}, u_{0}\right) \in X_{e_{0}}$ be an initial configuration with $h_{0}>0$, and let $T<T_{0}$. Then

(i) there exists a constant $C>0$ such that for all $N \in \mathbb{N}$ and $i=0, \ldots, N$,

$$
\left\|\nabla u_{i, N}\right\|_{C^{0, \frac{1}{2}}\left(\bar{\Omega}_{h_{i, N}} ; \mathbb{M}^{2 \times 2}\right)} \leq C,
$$

(ii) $E\left(u_{N}\right)\left(\cdot, h_{N}\right) \rightarrow E(u)(\cdot, h)$ in $C^{0, \beta}\left([0, T] ; C^{1, \alpha}([0, b])\right)$, 
(iii) $E\left(\tilde{u}_{N}\right)\left(\cdot, \tilde{h}_{N}\right) \rightarrow E(u)(\cdot, h)$ in $L^{\infty}\left(0, T ; C^{1, \alpha}([0, b])\right)$,

for every $\alpha \in\left(0, \frac{1}{2}\right)$ and $\beta \in\left(0, \frac{1-2 \alpha}{8}\right)$, where $u(\cdot, t)$ is the elastic equilibrium corresponding to $h(\cdot, t)$.

Proof. Recall that by Remark 3.4 we have $h_{N}, \tilde{h}_{N}>0$ in $[0, b] \times[0, T]$. Using standard elliptic estimates (see [21, Proposition 8.9]), for all $N \in \mathbb{N}$ and $i=0, \ldots, N$, we may bound the norm of $\nabla u_{i, N}$ in $C^{0, \frac{1}{2}}\left(\bar{\Omega}_{h_{i, N}} ; \mathbb{M}^{2 \times 2}\right)$ by a constant that depends only on the $C^{1, \frac{1}{2}}[0, b]$ norm of $h_{i, N}$ (and the fourth order tensor $\mathbb{C}$ ). Thus, the first assertion follows from the second estimate in (3.1).

In view of Lemma 5.6 and the second estimate in (3.1), the second and third assertions are implied by (3.8) and (3.19), respectively.

To simplify the notation, we define the function $W_{N}$ in $[0, b] \times(0, T]$ by $W_{N}(\cdot, t):=W_{i, N}$ for each $N \in \mathbb{N}$ and $t \in I_{i, N}$, where

$$
W_{i, N}(x):=W\left(E\left(u_{i, N}\right)\left(x, h_{i, N}(x)\right)\right),
$$

for each $i=1, \ldots, N$ and $x \in[0, b]$. Consider also, the function defined by $W(\cdot, t):=$ $W(E(u)(\cdot, h(\cdot, t)))$ in $[0, b]$ for each $t \in(0, T]$.

Theorem 3.8. Let $\left(h_{0}, u_{0}\right) \in X_{e_{0}}$ be an initial configuration that satisfies (3.21) and let $T<T_{0}$. Then

(i) there exists a constant $C>0$ such that for each $N \in \mathbb{N}$ we have

$$
\int_{0}^{T} \int_{0}^{b}\left|\frac{\partial^{4} \tilde{h}_{N}(x, t)}{\partial x^{4}}\right|^{2} \mathrm{~d} x \mathrm{~d} t \leq C
$$

(ii) $h \in L^{2}\left(0, T ; H^{4}(0, b)\right)$ and $\tilde{h}_{N} \rightarrow h$ in $L^{2}\left(0, T ; H^{4}(0, b)\right)$.

Proof. By Corollary 3.3, for all $N \in \mathbb{N}$ and $i=1, \ldots, N, h_{i, N}$ satisfies the Euler-Lagrange equation

$$
\int_{0}^{b}\left[\varepsilon \frac{h_{i, N}^{\prime \prime}}{J_{i, N}^{5}} \varphi^{\prime \prime}-\frac{5 \varepsilon}{2} \frac{\left(h_{i, N}^{\prime \prime}\right)^{2}}{J_{i, N}^{7}} h_{i, N}^{\prime} \varphi^{\prime}-\partial_{1} \psi\left(-h_{i, N}^{\prime}, 1\right) \varphi^{\prime}\right] \mathrm{d} x+\int_{0}^{b}\left(W_{i, N}+V_{i, N}\right) \varphi \mathrm{d} x=0
$$

for all $\varphi \in A P$, where $\partial_{1} \psi$ is the partial derivative of $\psi$ with respect to the first component and $W_{i, N}(x)$ is a continuous function in $[0, b]$ by Theorem 3.7. In particular, for all $N \in \mathbb{N}$, $i=1, \ldots, N$, and $\varphi \in C_{c}^{2}(0, b)$, we have that

$$
\int_{0}^{b} f_{i, N} \varphi^{\prime \prime} \mathrm{d} x=0
$$

where the function $f_{i, N}$, defined by

$f_{i, N}(x):=\varepsilon \frac{h_{i, N}^{\prime \prime}}{J_{i, N}^{5}}+\int_{0}^{x}\left(\frac{5 \varepsilon}{2} \frac{\left(h_{i, N}^{\prime \prime}\right)^{2}}{J_{i, N}^{7}} h_{i, N}^{\prime}+\partial_{1} \psi\left(-h_{i, N}^{\prime}, 1\right)\right) \mathrm{d} r+\int_{0}^{x} \int_{0}^{r}\left(W_{i, N}+V_{i, N}\right) \mathrm{d} \zeta \mathrm{d} r$, 
for $x \in[0, b]$, belongs to $L^{2}(0, b)$. Therefore, we conclude that

$$
f_{i, N}(x)=c_{i, N} x+d_{i, N}
$$

for every $x \in[0, b]$ and some constants $c_{i, N}$ and $d_{i, N}$. Now, solving (3.24) for $h_{i, N}^{\prime \prime}$, we obtain

$$
\begin{aligned}
h_{i, N}^{\prime \prime}=\frac{J_{i, N}^{5}}{\varepsilon}\left[-\int_{0}^{x}\left(\frac{5 \varepsilon}{2} \frac{\left(h_{i, N}^{\prime \prime}\right)^{2}}{J_{i, N}^{7}} h_{i, N}^{\prime}+\partial_{1} \psi\left(-h_{i, N}^{\prime}, 1\right)\right) \mathrm{d} r\right. \\
\left.\quad-\int_{0}^{x} \int_{0}^{r}\left(W_{i, N}+V_{i, N}\right) \mathrm{d} \zeta \mathrm{d} r+c_{i, N} x+d_{i, N}\right],
\end{aligned}
$$

from which we conclude that $h_{i, N}^{\prime \prime}$ is absolutely continuous on $[0, b]$, and so it is $b$-periodic (since $h_{i, N}$ is $b$-periodic). Furthermore, differentiating both side of (3.24) and solving the resulting equation for $h_{i, N}^{\prime \prime \prime}$, we obtain

$$
h_{i, N}^{\prime \prime \prime}=\frac{5}{2} \frac{\left(h_{i, N}^{\prime \prime}\right)^{2}}{J_{i, N}^{2}} h_{i, N}^{\prime}+\frac{J_{i, N}^{5}}{\varepsilon}\left(-\partial_{1} \psi\left(-h_{i, N}^{\prime}, 1\right)-\int_{0}^{x}\left(W_{i, N}+V_{i, N}\right) \mathrm{d} r+c_{i, N}\right) .
$$

Hence, $h_{i, N}^{\prime \prime \prime}$ is also absolutely continuous on $[0, b]$, and so it is $b$-periodic. Differentiating (3.24) once more and solving the resulting equation for $h_{i, N}^{(\mathrm{iv})}$, we obtain

$$
\begin{aligned}
h_{i, N}^{\text {(iv) }}=10 \frac{h_{i, N}^{\prime \prime \prime} h_{i, N}^{\prime \prime} h_{i, N}^{\prime}}{J_{i, N}^{2}}+ & \frac{5}{2} \frac{\left(h_{i, N}^{\prime \prime}\right)^{3}}{J_{i, N}^{2}}-\frac{35}{2} \frac{\left(h_{i, N}^{\prime \prime}\right)^{3}\left(h_{i, N}^{\prime}\right)^{2}}{J_{i, N}^{4}}+ \\
& +\frac{J_{i, N}^{5} h_{i, N}^{\prime \prime}}{\varepsilon} \partial_{11} \psi\left(-h_{i, N}^{\prime}, 1\right)-\frac{J_{i, N}^{5}}{\varepsilon}\left(W_{i, N}+V_{i, N}\right) .
\end{aligned}
$$

Thus, since $\psi$ is of class $C^{2}$ away from the origin, $h_{i, N} \in C^{4}([0, b])$, and so $h_{i, N} \in H_{\#}^{4}(0, b)$ with $h_{i, N}^{\text {(iv) }} b$-periodic. Furthermore, by Theorems 3.1 and 3.7, we have

$$
\begin{aligned}
\int_{0}^{b}\left|h_{i, N}^{(\mathrm{iv})}\right|^{2} \mathrm{~d} x & \leq C \int_{0}^{b}\left(1+\left|h_{i, N}^{\prime \prime}\right|^{6}+\left|h_{i, N}^{\prime \prime \prime}\right|^{2}\left|h_{i, N}^{\prime \prime}\right|^{2}+V_{i, N}^{2}\right) \mathrm{d} x \\
& \leq C \int_{0}^{b}\left|h_{i, N}^{\prime \prime}\right|^{6} \mathrm{~d} x+C \int_{0}^{b}\left|h_{i, N}^{\prime \prime \prime}\right|^{3} \mathrm{~d} x+C \int_{0}^{b}\left(1+V_{i, N}^{2}\right) \mathrm{d} x,
\end{aligned}
$$

where in the last inequality we used Young's inequality. Now we apply (5.7) and (5.8) to $h_{i, N}^{\prime \prime}$ to estimate $\left\|h_{i, N}^{\prime \prime}\right\|_{L^{6}(0, b)}$ and $\left\|h_{i, N}^{\prime \prime \prime}\right\|_{L^{3}(0, b)}$, respectively. It follows that

$$
\begin{aligned}
\left\|h_{i, N}^{(\mathrm{iv})}\right\|_{L^{2}}^{2} & \leq C\left\|h_{i, N}^{\prime \prime}\right\|_{L^{2}}^{5}\left\|h_{i, N}^{(\mathrm{iv})}\right\|_{L^{2}}+C\left\|h_{i, N}^{\prime \prime}\right\|_{L^{2}}^{\frac{5}{4}}\left\|h_{i, N}^{(\mathrm{iv})}\right\|_{L^{2}}^{\frac{7}{4}}+C \int_{0}^{b}\left(1+V_{i, N}^{2}\right) \mathrm{d} x \\
& \leq \gamma\left\|h_{i, N}^{(\mathrm{iv})}\right\|_{L^{2}(0, b)}^{2}+C_{\gamma} \int_{0}^{b}\left(1+V_{i, N}^{2}\right) \mathrm{d} x,
\end{aligned}
$$


where in the last inequality we used Young's inequality with an arbitrary $\gamma>0$ and (3.1) to estimate $\left\|h_{i, N}^{\prime \prime}\right\|_{L^{2}}$. Choosing $\gamma<1$ in (3.27), multiplying for $\frac{T}{N}$, and summing over all $i=1, \ldots, N$, we obtain

$$
\sum_{i=1}^{N} \frac{T}{N} \int_{0}^{b}\left|h_{i, N}^{(\mathrm{iv})}\right|^{2} \mathrm{~d} x \leq C \int_{0}^{T} \int_{0}^{b}\left(1+V_{N}^{2}\right) \mathrm{d} x \mathrm{~d} t .
$$

Hence, recalling the definition of $\tilde{h}_{N}$ since $V_{N}$ is bounded in $L^{2}\left(0, T ; L^{2}(0, b)\right)$ by $(3.20)$ we obtain (i).

We now prove the second assertion. We start by considering $M>N, i=1, \ldots, N$ and $j=1, \ldots, M$. Subtracting to (3.23) the Euler-Lagrange equation satisfied by $h_{j, M}$, and considering the test function $\varphi=h_{i, N}-h_{j, M}$, we obtain

$$
\begin{aligned}
\int_{0}^{b}\left(\frac{h_{i, N}^{\prime \prime}}{J_{i, N}^{5}}-\frac{h_{j, M}^{\prime \prime}}{J_{j, M}^{5}}\right)\left(h_{i, N}^{\prime \prime}-h_{j, M}^{\prime \prime}\right) \mathrm{d} & =\frac{5}{2} \int_{0}^{b}\left(\frac{\left(h_{i, N}^{\prime \prime}\right)^{2}}{J_{i, N}^{7}} h_{i, N}^{\prime}-\frac{\left(h_{j, M}^{\prime \prime}\right)^{2}}{J_{j, M}^{7}} h_{j, M}^{\prime}\right)\left(h_{i, N}^{\prime}-h_{j, M}^{\prime}\right) \mathrm{d} x \\
& +\frac{1}{\varepsilon} \int_{0}^{b}\left(\partial_{1} \psi\left(-h_{i, N}^{\prime}, 1\right)-\partial_{1} \psi\left(-h_{j, M}^{\prime}, 1\right)\right)\left(h_{i, N}^{\prime}-h_{j, M}^{\prime}\right) \mathrm{d} x \\
& -\frac{1}{\varepsilon} \int_{0}^{b}\left(W_{i, N}-W_{j, M}\right)\left(h_{i, N}-h_{j, M}\right) \mathrm{d} x \\
& -\frac{1}{\varepsilon} \int_{0}^{b}\left(V_{i, N}-V_{j, M}\right)\left(h_{i, N}-h_{j, M}\right) \mathrm{d} x .
\end{aligned}
$$

Fix $\eta>0$ and recall the notation $I_{i, N}=\left((i-1) \tau_{N}, i \tau_{N}\right]$ and $I_{j, M}=\left((j-1) \tau_{N}, j \tau_{N}\right]$. Since $\tilde{h}_{N} \rightarrow h$ in $L^{\infty}\left(0, T ; C^{1}([0, b])\right)$, for $N$ and $M$ sufficiently large and for every $i$ and $j$ such that $\left|I_{i, N} \cap I_{j, M}\right| \neq 0$, we have that $\left\|h_{i, N}-h_{j, M}\right\|_{C^{1}([0, b])} \leq \eta$. We claim that

$$
\int_{0}^{b}\left|h_{i, N}^{\prime \prime}-h_{j, M}^{\prime \prime}\right|^{2} d x \leq C \eta \int_{0}^{b}\left(1+\left|V_{i, N}\right|+\left|V_{j, M}\right|\right) \mathrm{d} x
$$

for some constant $C>0$. Indeed, the left-hand side of (3.28) satisfies

$$
\begin{aligned}
\mid \int_{0}^{b}\left(\frac{h_{i, N}^{\prime \prime}}{J_{i, N}^{5}}-\frac{h_{j, M}^{\prime \prime}}{J_{j, M}^{5}}\right) & \left(h_{i, N}^{\prime \prime}-h_{j, M}^{\prime \prime}\right) \mathrm{d} x \mid \\
& \geq \int_{0}^{b} \frac{\left|h_{i, N}^{\prime \prime}-h_{j, M}^{\prime \prime}\right|^{2}}{J_{i, N}^{5}} \mathrm{~d} x-\left|\int_{0}^{b} h_{j, M}^{\prime \prime}\left(\frac{1}{J_{j, M}^{5}}-\frac{1}{J_{i, N}^{5}}\right)\left(h_{i, N}^{\prime \prime}-h_{j, M}^{\prime \prime}\right) \mathrm{d} x\right| \\
& \geq C \int_{0}^{b}\left|h_{i, N}^{\prime \prime}-h_{j, M}^{\prime \prime}\right|^{2} \mathrm{~d} x-\int_{0}^{b}\left|\frac{1}{J_{j, M}^{5}}-\frac{1}{J_{i, N}^{5}}\right|\left|h_{j, M}^{\prime \prime}\right|\left(\left|h_{i, N}^{\prime \prime}\right|+\left|h_{j, M}^{\prime \prime}\right|\right) \mathrm{d} x \\
& \geq C \int_{0}^{b}\left|h_{i, N}^{\prime \prime}-h_{j, M}^{\prime \prime}\right|^{2} \mathrm{~d} x-C \eta
\end{aligned}
$$

where we used the Lipschitz continuity of the function $s \mapsto\left(1+s^{2}\right)^{-\frac{5}{2}}$ on $\left[0, r_{0}\right], J_{i, N} \leq$ $\sqrt{1+r_{0}^{2}}$, and (3.6). Thus, the claim follows from the fact that the absolute value of the 
right-hand side may be estimated from above by $C \eta$ for some constant $C>0$, since $h_{i, N}$, $h_{j, M} \leq r_{0},(3.6), \partial_{1} \psi$ is continuous away from the origin, and in view of assertion (iii) of Theorem 3.7.

Furthermore, integrating (3.29) over $I_{i, N} \cap I_{j, M}$, we have that for $N$ and $M$ sufficiently large,

$$
\begin{aligned}
\int_{I_{i, N} \cap I_{j, M}} \int_{0}^{b} \mid \frac{\partial^{2} \tilde{h}_{N}}{\partial x^{2}}(x, t)- & \left.\frac{\partial^{2} \tilde{h}_{M}}{\partial x^{2}}(x, t)\right|^{2} \mathrm{~d} x \mathrm{~d} t \\
& \leq C \eta \int_{I_{i, N} \cap I_{j, M}} \int_{0}^{b}\left(1+\left|V_{i, N}\right|+\left|V_{j, M}\right|\right) \mathrm{d} x \mathrm{~d} t
\end{aligned}
$$

for each $i$ and $j$ such that $\left|I_{i, N} \cap I_{j, M}\right| \neq 0$. Now, we first fix $i=1, \ldots, N$, and sum the previous estimate with respect to every $j$ such that $\left|I_{i, N} \cap I_{j, M}\right| \neq 0$ to obtain

$$
\begin{aligned}
\int_{I_{i, N}} \int_{0}^{b} \mid \frac{\partial^{2} \tilde{h}_{N}}{\partial x^{2}}(x, t)- & \left.\frac{\partial^{2} \tilde{h}_{M}}{\partial x^{2}}(x, t)\right|^{2} \mathrm{~d} x \mathrm{~d} t \\
& \leq C \eta \int_{I_{i, N}} \int_{0}^{b}\left(1+\left|V_{N}\right|+\left|V_{M}\right|\right) \mathrm{d} x \mathrm{~d} t,
\end{aligned}
$$

and then we sum over $i$, so that (3.20) implies

$$
\int_{0}^{T} \int_{0}^{b}\left|\frac{\partial^{2} \tilde{h}_{N}}{\partial x^{2}}(x, t)-\frac{\partial^{2} \tilde{h}_{M}}{\partial x^{2}}(x, t)\right|^{2} \mathrm{~d} x \mathrm{~d} t \leq C \eta
$$

for $M, N$ sufficiently large and some constant $C>0$.

Moreover, by (5.6),

$$
\begin{aligned}
& \int_{0}^{b}\left|\frac{\partial^{3} \tilde{h}_{N}}{\partial x^{3}}(x, t)-\frac{\partial^{3} \tilde{h}_{M}}{\partial x^{3}}(x, t)\right|^{2} \mathrm{~d} x \\
& \quad \leq C\left(\int_{0}^{b}\left|\frac{\partial^{4} \tilde{h}_{N}}{\partial x^{4}}(x, t)-\frac{\partial^{4} \tilde{h}_{M}}{\partial x^{4}}(x, t)\right|^{2} \mathrm{~d} x\right)^{\frac{1}{2}}\left(\int_{0}^{b}\left|\frac{\partial^{2} \tilde{h}_{N}}{\partial x^{2}}(x, t)-\frac{\partial^{2} \tilde{h}_{M}}{\partial x^{2}}(x, t)\right|^{2} \mathrm{~d} x\right)^{\frac{1}{2}} .
\end{aligned}
$$

Finally, we integrate with respect to $t$ and use Hölder's inequality, the first assertion and (3.30) to deduce that

$$
\int_{0}^{T} \int_{0}^{b}\left|\frac{\partial^{3} \tilde{h}_{N}}{\partial x^{3}}(x, t)-\frac{\partial^{3} \tilde{h}_{M}}{\partial x^{3}}(x, t)\right|^{2} \mathrm{~d} x \mathrm{~d} t \leq C \eta^{\frac{1}{2}},
$$

for $N$ and $M$ sufficiently large. Thus, by (3.30) and (3.31), $\left\{\frac{\partial^{2} \tilde{h}_{N}}{\partial x^{2}}\right\}$ is a Cauchy sequence in $L^{2}\left(0, T ; H^{1}(0, b)\right)$ and, since by Theorem 3.1 and $(3.19) \tilde{h}_{N} \rightarrow h$ in $L^{2}\left(0, T ; H^{2}(0, b)\right)$, we have that $\tilde{h}_{N} \rightarrow h$ in $L^{2}\left(0, T ; H^{3}(0, b)\right)$. Hence, in view of (i) we obtain that $\tilde{h}_{N} \rightarrow h$ in $L^{2}\left(0, T ; H^{4}(0, b)\right)$. 
Note that $h \in L^{2}\left(0, T ; H_{\#}^{4}(0, b)\right) \cap H^{1}\left(0, T ; L_{\#}^{2}(0, b)\right)$ and recall Definition 2.6. In the following theorem, we prove the existence of a solution of (1.10) in $[0, T]$ for $T<T_{0}$.

Theorem 3.9. Let $\left(h_{0}, u_{0}\right) \in X_{e_{0}}$ be an initial configuration such that $h_{0}>0$, and let $T_{0}>0$ be as defined in (3.17). Then the Cauchy problem (1.10) admits a solution in $[0, T]$ for each $T<T_{0}$ in the sense of Definition 2.6.

Proof. Fix $\varphi \in C_{c}^{\infty}((0, b) \times(0, T))$. It follows from (3.23) that for all $N \in \mathbb{N}$,

$$
\int_{0}^{b}\left[\varepsilon \frac{\left(\tilde{h}_{N}\right)_{x x}}{\tilde{J}_{N}^{5}} \varphi_{x x}-\frac{5 \varepsilon}{2} \frac{\left(\tilde{h}_{N}\right)_{x x}^{2}}{\tilde{J}_{N}^{7}}\left(\tilde{h}_{N}\right)_{x} \varphi_{x}-\partial_{1} \psi\left(-\left(\tilde{h}_{N}\right)_{x}, 1\right) \varphi_{x}+W_{N} \varphi\right] \mathrm{d} x=-\int_{0}^{b} V_{N} \varphi \mathrm{d} x
$$

in $(0, T]$. Integrating over $(0, T]$, we obtain

$$
\int_{0}^{T} A_{N} \mathrm{~d} t=-\int_{0}^{T} \int_{0}^{b} V_{N} \varphi \mathrm{d} x \mathrm{~d} t
$$

where

$$
A_{N}:=\int_{0}^{b}\left[\varepsilon \frac{\left(\tilde{h}_{N}\right)_{x x}}{\tilde{J}_{N}^{5}} \varphi_{x x}-\frac{5 \varepsilon}{2} \frac{\left(\tilde{h}_{N}\right)_{x x}^{2}}{\tilde{J}_{N}^{7}}\left(\tilde{h}_{N}\right)_{x} \varphi_{x}-\partial_{1} \psi\left(-\left(\tilde{h}_{N}\right)_{x}, 1\right) \varphi_{x}+W_{N} \varphi\right] \mathrm{d} x
$$

in $(0, T]$. By Lebesgue Dominated Convergence Theorem, $\left\{A_{N}\right\}$ converges to

$$
A:=\int_{0}^{b}\left[\varepsilon \frac{h_{x x}}{J^{5}} \varphi_{x x}-\frac{5 \varepsilon}{2} \frac{h_{x x}^{2}}{J^{7}} h_{x} \varphi_{x}-\partial_{1} \psi\left(-h_{x}, 1\right) \varphi_{x}+W \varphi\right] \mathrm{d} x
$$

in $L^{1}(0, T)$. Indeed, we have that

$$
\left|A_{N}\right| \leq C\|\varphi\|_{C^{2}((0, b) \times(0, T))} \int_{0}^{b}\left[\left|\left(\tilde{h}_{N}\right)_{x x}\right|+\left|\left(\tilde{h}_{N}\right)_{x x}\right|^{2}+W_{N}\right] \mathrm{d} x
$$

in $(0, T]$ for some constant $C>0$, since $\left(\tilde{h}_{N}\right)_{x}$ is uniformly bounded in $[0, b] \times(0, T]$, $\partial_{1} \psi$ is continuous away from the origin, and $\tilde{J}_{N} \geq 1$. Thus, by (3.1) and assertion (i) of Theorem 3.7, $A_{N}$ is uniformly bounded in $(0, T]$. Moreover, $A_{N} \rightarrow A \mathcal{L}^{1}$-a.e. in $(0, T)$ because $\partial_{1} \psi$ is continuous away from the origin, $W_{N}(\cdot, t) \rightarrow W(\cdot, t)$ in $C([0, b])$ by Theorem 3.7 , and $\tilde{h}_{N}(\cdot, t) \rightarrow h(\cdot, t)$ in $C^{2}([0, b])$ by Theorem 3.8 .

Therefore, since $A_{N} \rightarrow A$ in $L^{1}(0, T)$ and also by (3.20), we obtain that

$$
\int_{0}^{T} \int_{0}^{b}\left[\varepsilon \frac{h_{x x}}{J^{5}} \varphi_{x x}-\frac{5 \varepsilon}{2} \frac{h_{x x}^{2}}{J^{7}} h_{x} \varphi_{x}-\partial_{1} \psi\left(-h_{x}, 1\right) \varphi_{x}+W \varphi\right] \mathrm{d} x \mathrm{~d} t=-\int_{0}^{T} \int_{0}^{b} V \varphi \mathrm{d} x \mathrm{~d} t .
$$

Integrating by parts, we have

$$
\int_{0}^{T} \int_{0}^{b} f \varphi \mathrm{d} x \mathrm{~d} t=0
$$

where the function $f$ defined in $[0, b] \times(0, T)$ by

$$
f:=\varepsilon\left(\frac{h_{x x}}{J^{5}}\right)_{x x}+\frac{5 \varepsilon}{2}\left(\frac{h_{x x}^{2}}{J^{7}} h_{x}\right)_{x}+\left(\partial_{1} \psi\left(-h_{x}, 1\right)\right)_{x}+W+V,
$$


belongs to $L^{2}\left(0, T ; L^{2}(0, b)\right)$. Indeed, since $h_{x}$ is uniformly bounded in $[0, b] \times[0, T], J \geq 1$, and $\partial_{11} \psi$ is continuous away from the origin, we have

$$
\begin{aligned}
\int_{0}^{T} \int_{0}^{b}|f|^{2} \mathrm{~d} x \mathrm{~d} t & \leq C \int_{0}^{T} \int_{0}^{b}\left[\left|h_{x x x x}\right|^{2}+\left|h_{x x x}\right|^{2}\left|h_{x x}\right|^{2}+\left|h_{x x}\right|^{6}+\left|h_{x x}\right|^{2}+W^{2}+|V|^{2}\right] \mathrm{d} x \mathrm{~d} t \\
& \leq C \int_{0}^{T} \int_{0}^{b}\left[1+\left|h_{x x x}\right|^{2}\left|h_{x x}\right|^{2}+\left|h_{x x}\right|^{6}\right] \mathrm{d} x \mathrm{~d} t \\
& \leq C \int_{0}^{T} \int_{0}^{b}\left[1+\left|h_{x x x}\right|^{3}+\left|h_{x x}\right|^{6}\right] \mathrm{d} x \mathrm{~d} t
\end{aligned}
$$

for some constant $C>0$, where in the second inequality we used the fact that $h$ belongs to $L^{2}\left(0, T_{0} ; H^{4}(0, b)\right),(3.20)$ and Theorem 3.7, while the last one follows from Young's inequality. Moreover, since $h_{x x}(\cdot, t) \in H_{\#}^{2}(0, b)$ for $\mathcal{L}^{1}$-a.e. $t$ in $\left[0, T_{0}\right]$, we may use the interpolation results $(5.7)$ and $(5.8)$ to estimate $\left\|h_{x x x}(\cdot, t)\right\|_{L^{3}(0, b)}$ and $\left\|h_{x x}(\cdot, t)\right\|_{L^{6}(0, b)}$, respectively, as done in (3.27), and then applying again Young's inequality, we obtain

$$
\int_{0}^{T} \int_{0}^{b}|f|^{2} \mathrm{~d} x \mathrm{~d} t \leq C\left[1+\int_{0}^{T} \int_{0}^{b}\left|h_{x x x x}\right|^{2} \mathrm{~d} x \mathrm{~d} t+\int_{0}^{T}\left(\int_{0}^{b}\left|h_{x x}\right|^{2} \mathrm{~d} x\right)^{5} \mathrm{~d} t\right] .
$$

Note that since $h \in L^{2}\left(0, T ; H^{4}(0, b)\right) \cap L^{\infty}\left(0, T ; H^{2}(0, b)\right)$, the right-hand side of the previous inequality is bounded.

By the arbitrariness of $\varphi$ and the density of $C_{c}^{\infty}((0, b) \times(0, T))$ in $L^{2}((0, b) \times(0, T))$, we deduce from (3.33) that $f \equiv 0$. Thus, $h$ satisfies

$$
V=-\varepsilon\left(\frac{h_{x x}}{J^{5}}\right)_{x x}-\frac{5 \varepsilon}{2}\left(\frac{h_{x x}^{2}}{J^{7}} h_{x}\right)_{x}-\left(\partial_{1} \psi\left(-h_{x}, 1\right)\right)_{x}-W
$$

which is $(2.15)$.

The following regularity result applies to the solution $h$ of (1.10) for $T<T_{0}$.

Theorem 3.10. Let $\left(h_{0}, u_{0}\right) \in X_{e_{0}}$ be an initial configuration such that $h_{0}>0$ and let $T<T_{0}$. Then, the solution $h$ of (1.10) in $[0, T]$ given in Theorem 3.9, satisfies:

(i) $h \in L^{2}\left(0, T ; H_{\#}^{4}(0, b)\right) \cap L^{\infty}\left(0, T ; H_{\#}^{2}(0, b)\right) \cap H^{1}\left(0, T ; L_{\#}^{2}(0, b)\right)$,

(ii) $h \in C^{0, \beta}\left([0, T] ; C^{1, \alpha}([0, b])\right)$ for every $\alpha \in\left(0, \frac{1}{2}\right)$ and $\beta \in\left(0, \frac{1-2 \alpha}{8}\right)$,

(iii) $\left\|h_{x}\right\|_{L^{\infty}\left(0, T ; L^{\infty}(0, b)\right)} \leq\left\|h_{0}^{\prime}\right\|_{\infty}+\sqrt{\left\|h_{0}^{\prime}\right\|_{\infty}^{2}+1}$,

(iv) $h \in L^{\frac{12}{5}}\left(0, T ; C_{\#}^{2,1}([0, b])\right) \cap L^{\frac{24}{5}}\left(0, T ; C_{\#}^{1,1}([0, b])\right)$.

Proof. Properties (i)-(iii) have been established in Theorems 3.1, 3.2, 3.8, and Corollary 3.3. In order to prove (iv), we fix $N, M \in \mathbb{N}$ and we follow [19, Corollary 3.7]. By (5.8), we 
have

$$
\begin{aligned}
& \left\|\frac{\partial^{3} \tilde{h}_{N}}{\partial x^{3}}(\cdot, t)-\frac{\partial^{3} \tilde{h}_{M}}{\partial x^{3}}(\cdot, t)\right\|_{\infty} \\
& \quad \leq C\left(\int_{0}^{b}\left|\frac{\partial^{4} \tilde{h}_{N}}{\partial x^{4}}(x, t)-\frac{\partial^{4} \tilde{h}_{M}}{\partial x^{4}}(x, t)\right|^{2} \mathrm{~d} x\right)^{\frac{5}{12}}\left(\int_{0}^{b}\left|\frac{\partial \tilde{h}_{N}}{\partial x}(x, t)-\frac{\partial \tilde{h}_{M}}{\partial x}(x, t)\right|^{2} \mathrm{~d} x\right)^{\frac{1}{12}}
\end{aligned}
$$

$\mathcal{L}^{1}$-a.e. in $[0, T]$. Raising both sides to the power $\frac{12}{5}$, integrating over $[0, T]$ and recalling (3.22), we obtain

$$
\int_{0}^{T}\left\|\frac{\partial^{3} \tilde{h}_{N}}{\partial x^{3}}(\cdot, t)-\frac{\partial^{3} \tilde{h}_{M}}{\partial x^{3}}(\cdot, t)\right\|_{\infty}^{\frac{12}{5}} \mathrm{~d} t \leq C \sup _{t \in[0, T]}\left\|\frac{\partial \tilde{h}_{N}}{\partial x}(\cdot, t)-\frac{\partial \tilde{h}_{M}}{\partial x}(\cdot, t)\right\|_{\infty}^{\frac{2}{5}} .
$$

Then, by (3.19) we have that $\tilde{h}_{N} \rightarrow h$ in $L^{\frac{12}{5}}\left(0, T ; C_{\#}^{2,1}([0, b])\right)$ and $h \in L^{\frac{12}{5}}\left(0, T ; C_{\#}^{2,1}([0, b])\right)$. Furthermore, by (5.6), we have

$$
\left\|\frac{\partial^{2} \tilde{h}_{N}}{\partial x^{2}}(\cdot, t)-\frac{\partial^{2} \tilde{h}_{M}}{\partial x^{2}}(\cdot, t)\right\|_{\infty} \leq C\left\|\frac{\partial^{3} \tilde{h}_{N}}{\partial x^{3}}(\cdot, t)-\frac{\partial^{3} \tilde{h}_{M}}{\partial x^{3}}(\cdot, t)\right\|_{\infty}^{\frac{1}{2}}\left\|\frac{\partial \tilde{h}_{N}}{\partial x}(\cdot, t)-\frac{\partial \tilde{h}_{M}}{\partial x}(\cdot, t)\right\|_{\infty}^{\frac{1}{2}}
$$

$\mathcal{L}^{1}$-a.e. in $[0, T]$. Thus, raising both sides to the power $\frac{24}{5}$, we proceed as before to conclude that $\tilde{h}_{N} \rightarrow h$ in $L^{\frac{24}{5}}\left(0, T ; C_{\#}^{1,1}([0, b])\right)$ and $h \in L^{\frac{24}{5}}\left(0, T ; C_{\#}^{1,1}([0, b])\right)$.

\section{UNIQUENESS}

From Theorem 4.1 below, it follows that the solution provided by Theorem 3.9 is the unique solution of $(1.10)$ in $[0, T]$ for $T<T_{0}$. Since (2.15) does not necessarily preserve the area underneath the profile of the film, the proof is more involved than the one in [19] for the case with surface diffusion.

Theorem 4.1. Let $\left(h_{0}, u_{0}\right) \in X_{e_{0}}$ be an initial configuration such that $h_{0}>0$, and let $T>0$. If $h_{1}, h_{2} \in L^{2}\left(0, T ; H_{\#}^{4}(0, b)\right) \cap L^{\infty}\left(0, T ; H_{\#}^{2}(0, b)\right) \cap H^{1}\left(0, T ; L_{\#}^{2}(0, b)\right)$ are two solutions of (1.10) in $[0, T]$ with initial configuration $\left(h_{0}, u_{0}\right)$ (see Definition 2.6), then $h_{1}=h_{2}$.

Proof. For simplicity of notation, in this proof, we denote by $(\cdot)^{\prime}$ the differentiation with respect to $x$. Consider a constant $M>0$ such that

$$
\left\|h_{i}\right\|_{L^{\infty}\left(0, T ; H_{\#}^{2}(0, b)\right)} \leq M
$$

for $i=1,2$. We want to apply Gronwall's Lemma to the function

$$
t \mapsto H(t):=\int_{0}^{b}\left|h_{2}-h_{1}\right|^{2} \mathrm{~d} x+\int_{0}^{b}\left|h_{2}^{\prime}-h_{1}^{\prime}\right|^{2} \mathrm{~d} x .
$$


We claim that $H \in W^{1,1}(0, T)$, and that there exists a constant $C>0$, that depends only on $M$, such that

$$
\frac{\partial H}{\partial t}(t) \leq C G(t) H(t)
$$

for almost every $t \in(0, T)$, where

$$
G(t):=1+\left\|h_{1}^{(\mathrm{iv})}(\cdot, t)\right\|_{L^{2}}^{2}+\left\|h_{2}^{(\mathrm{iv})}(\cdot, t)\right\|_{L^{2}}^{2} .
$$

We proceed in four steps. In the sequel of this proof, constants denoted by the same symbol may change from formula to formula.

Step 1: We begin by proving that $H \in W^{1,1}(0, T)$, and that for almost every $t \in(0, T)$, we have

$$
\frac{1}{2} \frac{\partial}{\partial t} \int_{0}^{b}\left|h_{2}-h_{1}\right|^{2} \mathrm{~d} x=\int_{0}^{b}\left(\frac{\partial h_{2}}{\partial t}-\frac{\partial h_{1}}{\partial t}\right)\left(h_{2}-h_{1}\right) \mathrm{d} x,
$$

and

$$
\frac{1}{2} \frac{\partial}{\partial t} \int_{0}^{b}\left|h_{2}^{\prime}-h_{1}^{\prime}\right|^{2} \mathrm{~d} x=-\int_{0}^{b}\left(\frac{\partial h_{2}}{\partial t}-\frac{\partial h_{1}}{\partial t}\right)\left(h_{2}^{\prime \prime}-h_{1}^{\prime \prime}\right) \mathrm{d} x
$$

To this purpose, we mollify the $b$-periodic function $\bar{h}$ defined in $\mathbb{R} \times(-T, 2 T)$ by

$$
\bar{h}(\cdot, t):= \begin{cases}\left(h_{2}-h_{1}\right)(\cdot, t) & \text { if } t \in[0, T], \\ \left(h_{2}-h_{1}\right)(\cdot,-t) & \text { if } t \in(-T, 0), \\ \left(h_{2}-h_{1}\right)(\cdot, 2 T-t) & \text { if } t \in(T, 2 T) .\end{cases}
$$

For each $\epsilon>0$ small enough, the mollification $\bar{h}_{\epsilon}$ is defined and smooth in $\mathbb{R} \times[0, T]$ and so, it satisfies

$$
\frac{1}{2} \frac{\partial}{\partial t} \int_{0}^{b}\left|\bar{h}_{\epsilon}\right|^{2} \mathrm{~d} x=\int_{0}^{b} \frac{\partial \bar{h}_{\epsilon}}{\partial t} \bar{h}_{\epsilon} \mathrm{d} x \quad \text { and } \quad \frac{1}{2} \frac{\partial}{\partial t} \int_{0}^{b}\left|\bar{h}_{\epsilon}^{\prime}\right|^{2} \mathrm{~d} x=-\int_{0}^{b} \frac{\partial \bar{h}_{\epsilon}}{\partial t} \bar{h}_{\epsilon}^{\prime \prime} \mathrm{d} x
$$

in $[0, T]$, where we used the fact that $\bar{h}_{\epsilon}(\cdot, t)$ is $b$-periodic for each $t \in[0, T]$. Furthermore, $\bar{h}_{\epsilon} \rightarrow \bar{h}$ in $H^{1}((0, b) \times(0, T))$ since $\bar{h} \in H^{1}((-b, 2 b) \times(-T, 2 T))$, and $\bar{h}_{\epsilon}^{\prime \prime} \rightarrow \bar{h}^{\prime \prime}$ in $L^{2}((0, b) \times$ $(0, T))$ since $\bar{h}^{\prime \prime} \in L^{2}((-b, 2 b) \times(-T, 2 T))$ (see [29]). Therefore, by (4.5) we obtain that $\int_{0}^{b}|\bar{h}|^{2} \mathrm{~d} x$ and $\int_{0}^{b}\left|\bar{h}^{\prime}\right|^{2} \mathrm{~d} x$ are weakly differentiable in the sense of distributions in $(0, T)$ and satisfy (4.3) and (4.4), respectively. 
Step 2: Inserting (2.15) for $h_{1}$ and $h_{2}$ in (4.3), integrating by parts, and using the periodicity of $h_{1}(\cdot, t)$ and $h_{2}(\cdot, t)$, we obtain

$$
\begin{aligned}
\frac{1}{2} \frac{\partial}{\partial t} \int_{0}^{b}\left|h_{2}-h_{1}\right|^{2} \mathrm{~d} x= & -\varepsilon \int_{0}^{b}\left[\frac{h_{2}^{\prime \prime}}{J_{2}^{5}}\left(J_{2}\left(h_{2}-h_{1}\right)\right)^{\prime \prime}-\frac{h_{1}^{\prime \prime}}{J_{1}^{5}}\left(J_{1}\left(h_{2}-h_{1}\right)\right)^{\prime \prime}\right] \mathrm{d} x \\
& +\frac{5 \varepsilon}{2} \int_{0}^{b}\left[\frac{\left(h_{2}^{\prime \prime}\right)^{2} h_{2}^{\prime}}{J_{2}^{7}}\left(J_{2}\left(h_{2}-h_{1}\right)\right)^{\prime}-\frac{\left(h_{1}^{\prime \prime}\right)^{2} h_{1}^{\prime}}{J_{1}^{7}}\left(J_{1}\left(h_{2}-h_{1}\right)\right)^{\prime}\right] \mathrm{d} x \\
& +\int_{0}^{b} \partial_{1} \psi\left(-h_{2}^{\prime}, 1\right)\left(J_{2}\left(h_{2}-h_{1}\right)\right)^{\prime}-\partial_{1} \psi\left(-h_{1}^{\prime}, 1\right)\left(J_{1}\left(h_{2}-h_{1}\right)\right)^{\prime} \mathrm{d} x \\
& -\int_{0}^{b}\left(W_{2} J_{2}-W_{1} J_{1}\right)\left(h_{2}-h_{1}\right) \mathrm{d} x=: I_{1}+I_{2}+I_{3}+I_{4},
\end{aligned}
$$

where $J_{i}$ and $W_{i}$ refer to the function $h_{i}$ for $i=1,2$. In the sequel of this step, we estimate the integrals on the right-hand side of the previous equality.

First, we consider $I_{1}$ and $I_{2}$ and observe that

$$
\begin{aligned}
& I_{1}+I_{2}+\varepsilon \int_{0}^{b} \frac{\left|h_{2}^{\prime \prime}-h_{1}^{\prime \prime}\right|^{2}}{J_{2}^{4}} \mathrm{~d} x= \varepsilon \int_{0}^{b} h_{1}^{\prime \prime}\left(\frac{1}{J_{2}^{4}}-\frac{1}{J_{1}^{4}}\right)\left(h_{2}^{\prime \prime}-h_{1}^{\prime \prime}\right) \mathrm{d} x \\
&+\frac{3 \varepsilon}{2} \int_{0}^{b}\left(\frac{\left(h_{2}^{\prime \prime}\right)^{2} h_{2}^{\prime}}{J_{2}^{6}}-\frac{\left(h_{1}^{\prime \prime}\right)^{2} h_{1}^{\prime}}{J_{1}^{6}}\right)\left(h_{2}^{\prime}-h_{1}^{\prime}\right) \mathrm{d} x \\
&+\frac{5 \varepsilon}{2} \int_{0}^{b}\left(\frac{\left(h_{2}^{\prime \prime \prime}\right)^{3}\left(h_{2}^{\prime}\right)^{2}}{J_{2}^{8}}-\frac{\left(h_{1}^{\prime \prime \prime}\right)^{3}\left(h_{1}^{\prime}\right)^{2}}{J_{1}^{8}}\right)\left(h_{2}-h_{1}\right) \mathrm{d} x \\
&-\int_{0}^{b}\left(\frac{\left(h_{2}^{\prime \prime}\right)^{3}+h_{2}^{\prime \prime \prime} h_{2}^{\prime \prime} h_{2}^{\prime}+h_{2}^{\prime \prime \prime} h_{2}^{\prime \prime}\left(h_{2}^{\prime}\right)^{3}}{J_{2}^{8}}-\frac{\left(h_{1}^{\prime \prime}\right)^{3}+h_{1}^{\prime \prime \prime} h_{1}^{\prime \prime} h_{1}^{\prime}+h_{1}^{\prime \prime \prime} h_{1}^{\prime \prime}\left(h_{1}^{\prime}\right)^{3}}{J_{1}^{8}}\right)\left(h_{2}-h_{1}\right) \mathrm{d} x .
\end{aligned}
$$

In view of (4.1), $h_{1}^{\prime}$ and $h_{2}^{\prime}$ are uniformly bounded and so there exists a constant $C_{\varepsilon}>0$ that depends on $M$ such that

$$
\inf _{(0, b) \times(0, T)} \frac{\varepsilon}{J_{2}^{4}} \geq C_{\varepsilon}
$$

Thus, since for $n \in \mathbb{N}$ the function $s \mapsto\left(1+s^{2}\right)^{-\frac{n}{2}}$ is locally Lipschitz continuous and we have

$$
\left|\left(h_{2}^{\prime \prime}\right)^{n}-\left(h_{1}^{\prime \prime}\right)^{n}\right| \leq\left(\left\|h_{1}^{\prime \prime}\right\|_{\infty}^{n-1}+\left\|h_{2}^{\prime \prime}\right\|_{\infty}^{n-1}\right)\left|h_{2}^{\prime \prime}-h_{1}^{\prime \prime}\right|
$$


in $(0, b) \times(0, T)$, we obtain

$$
\begin{aligned}
I_{1}+ & I_{2}+C_{\varepsilon} \int_{0}^{b}\left|h_{2}^{\prime \prime}-h_{1}^{\prime \prime}\right|^{2} \mathrm{~d} x \leq C\left[\int_{0}^{b}\left|h_{1}^{\prime \prime}\right|\left|h_{2}^{\prime \prime}-h_{1}^{\prime \prime}\right|\left|h_{2}^{\prime}-h_{1}^{\prime}\right| \mathrm{d} x+\int_{0}^{b}\left|h_{2}^{\prime \prime}\right|^{2}\left|h_{2}^{\prime}-h_{1}^{\prime}\right|^{2} \mathrm{~d} x\right. \\
& +\left(\left\|h_{1}^{\prime \prime}\right\|_{\infty}+\left\|h_{2}^{\prime \prime}\right\|_{\infty}\right) \int_{0}^{b}\left|h_{2}^{\prime \prime}-h_{1}^{\prime \prime}\right|\left|h_{2}^{\prime}-h_{1}^{\prime}\right| \mathrm{d} x+\int_{0}^{b}\left(\left|h_{2}^{\prime \prime}\right|\left|h_{2}^{\prime}-h_{1}^{\prime}\right|\right)\left(\left|h_{2}^{\prime \prime}\right|^{2}\left|h_{2}-h_{1}\right|\right) \mathrm{d} x \\
& +\left(\left\|h_{1}^{\prime \prime}\right\|_{\infty}^{2}+\left\|h_{2}^{\prime \prime}\right\|_{\infty}^{2}\right) \int_{0}^{b}\left|h_{2}^{\prime \prime}-h_{1}^{\prime \prime}\right|\left|h_{2}-h_{1}\right| \mathrm{d} x+\int_{0}^{b}\left(\left|h_{2}^{\prime \prime}\right|\left|h_{2}^{\prime}-h_{1}^{\prime}\right|\right)\left(\left|h_{2}^{\prime \prime \prime}\right|\left|h_{2}-h_{1}\right|\right) \mathrm{d} x \\
& \left.+\int_{0}^{b}\left|h_{2}^{\prime \prime}\right|\left|h_{2}^{\prime \prime \prime}-h_{1}^{\prime \prime \prime}\right|\left|h_{2}-h_{1}\right| \mathrm{d} x+\int_{0}^{b}\left|h_{1}^{\prime \prime \prime}\right|\left|h_{2}^{\prime \prime}-h_{1}^{\prime \prime}\right|\left|h_{2}-h_{1}\right| \mathrm{d} x\right] .
\end{aligned}
$$

We now apply Young's inequality to each integral on the right-hand side of the previous inequality. Precisely, for the integrals that present the term $\left|h_{2}^{\prime \prime}-h_{1}^{\prime \prime}\right|$ or $\left|h_{2}^{\prime \prime \prime}-h_{1}^{\prime \prime \prime}\right|$, we use Young's inequality with a parameter $\eta>0$. In this way, we have that

$$
\begin{array}{r}
I_{1}+I_{2}+C_{\varepsilon} \int_{0}^{b}\left|h_{2}^{\prime \prime}-h_{1}^{\prime \prime}\right|^{2} \mathrm{~d} x \leq \eta \int_{0}^{b}\left|h_{2}^{\prime \prime \prime}-h_{1}^{\prime \prime \prime}\right|^{2} \mathrm{~d} x+\eta \int_{0}^{b}\left|h_{2}^{\prime \prime}-h_{1}^{\prime \prime}\right|^{2} \mathrm{~d} x \\
+C_{\eta}\left(\left\|h_{1}^{\prime \prime}\right\|_{\infty}^{2}+\left\|h_{2}^{\prime \prime}\right\|_{\infty}^{2}\right) \int_{0}^{b}\left|h_{2}^{\prime}-h_{1}^{\prime}\right|^{2} \mathrm{~d} x \\
+C_{\eta}\left(\left\|h_{2}^{\prime \prime}\right\|_{\infty}^{2}+\left\|h_{1}^{\prime \prime}\right\|_{\infty}^{4}+\left\|h_{2}^{\prime \prime}\right\|_{\infty}^{4}+\left\|h_{1}^{\prime \prime \prime}\right\|_{\infty}^{2}+\left\|h_{2}^{\prime \prime \prime}\right\|_{\infty}^{2}\right) \int_{0}^{b}\left|h_{2}-h_{1}\right|^{2} \mathrm{~d} x
\end{array}
$$

Next, we estimate $I_{3}$ from above. As before, we begin by observing that

$$
\begin{aligned}
I_{3}= & \int_{0}^{b}\left(\partial_{1} \psi\left(-h_{2}^{\prime}, 1\right) J_{2}-\partial_{1} \psi\left(-h_{1}^{\prime}, 1\right) J_{1}\right)\left(h_{2}^{\prime}-h_{1}^{\prime}\right) \mathrm{d} x \\
& +\int_{0}^{b}\left(\partial_{1} \psi\left(-h_{2}^{\prime}, 1\right) \frac{h_{2}^{\prime \prime} h_{2}^{\prime}}{J_{2}}-\partial_{1} \psi\left(-h_{1}^{\prime}, 1\right) \frac{h_{1}^{\prime \prime} h_{1}^{\prime}}{J_{1}}\right)\left(h_{2}-h_{1}\right) \mathrm{d} x .
\end{aligned}
$$

Then, using the fact that the function $s \mapsto \partial_{1} \psi(s, 1)$ is locally Lipschitz continuous, and again invoking the fact that $h_{1}^{\prime}$ and $h_{2}^{\prime}$ are uniformly bounded, we have

$$
\begin{aligned}
I_{3} & \leq C\left[\int_{0}^{b}\left|h_{2}^{\prime}-h_{1}^{\prime}\right|^{2} \mathrm{~d} x+\int_{0}^{b}\left|h_{2}^{\prime \prime}\right|\left|h_{2}^{\prime}-h_{1}^{\prime}\right|\left|h_{2}-h_{1}\right| \mathrm{d} x+\int_{0}^{b}\left|h_{2}^{\prime \prime}-h_{1}^{\prime \prime}\right|\left|h_{2}-h_{1}\right| \mathrm{d} x\right] \\
& \leq \eta \int_{0}^{b}\left|h_{2}^{\prime \prime}-h_{1}^{\prime \prime}\right|^{2} \mathrm{~d} x+C \int_{0}^{b}\left|h_{2}^{\prime}-h_{1}^{\prime}\right|^{2} \mathrm{~d} x+C_{\eta}\left(1+\left\|h_{2}^{\prime \prime}\right\|_{\infty}^{2}\right) \int_{0}^{b}\left|h_{2}-h_{1}\right|^{2} \mathrm{~d} x .
\end{aligned}
$$

Now, we consider $I_{4}$. Observe that by Lemma 5.6 and by the definition of $W$, there exists a constant $C$, that depends on $M$, such that $\left\|W_{i}\right\|_{L^{\infty}((0, b) \times(0, T))} \leq C$ for $i=1,2$, and

$$
\int_{0}^{b}\left|W_{2}-W_{1}\right|^{2} \mathrm{~d} x \leq C\left\|h_{1}-h_{2}\right\|_{H^{2}}^{2} \leq C \int_{0}^{b}\left|h_{2}-h_{1}\right|^{2} \mathrm{~d} x+C \int_{0}^{b}\left|h_{2}^{\prime \prime}-h_{1}^{\prime \prime}\right|^{2} \mathrm{~d} x
$$

in $(0, T)$, where in the last estimate we applied Poincaré inequality. Therefore, since the function $s \mapsto\left(1+s^{2}\right)^{\frac{1}{2}}$ is locally Lipschitz continuous, $W_{i}$ and $h_{i}^{\prime}$ are uniformly bounded 
for $i=1,2$, we have

$$
\begin{aligned}
I_{4} & :=-\int_{0}^{b}\left(W_{2} J_{2}-W_{1} J_{1}\right)\left(h_{2}-h_{1}\right) \mathrm{d} x \\
& \leq C \int_{0}^{b}\left|W_{2}-W_{1}\right|\left|h_{2}-h_{1}\right| \mathrm{d} x+C \int_{0}^{b}\left|h_{2}^{\prime}-h_{1}^{\prime}\right|\left|h_{2}-h_{1}\right| \mathrm{d} x \\
& \leq \eta \int_{0}^{b}\left|W_{2}-W_{1}\right|^{2} \mathrm{~d} x+C \int_{0}^{b}\left|h_{2}^{\prime}-h_{1}^{\prime}\right|^{2} \mathrm{~d} x+C_{\eta} \int_{0}^{b}\left|h_{2}-h_{1}\right|^{2} \mathrm{~d} x \\
& \leq \eta \int_{0}^{b}\left|h_{2}^{\prime \prime}-h_{1}^{\prime \prime}\right|^{2} \mathrm{~d} x+C \int_{0}^{b}\left|h_{2}^{\prime}-h_{1}^{\prime}\right|^{2} \mathrm{~d} x+C_{\eta} \int_{0}^{b}\left|h_{2}-h_{1}\right|^{2} \mathrm{~d} x,
\end{aligned}
$$

where in the second inequality we used Young's inequality (with and without a small parameter $\eta>0$ ), while in the last we used (4.11).

Finally, combining (4.9), (4.10) and (4.12) with (4.6), we obtain that

$$
\begin{aligned}
& \frac{\partial}{\partial t} \int_{0}^{b}\left|h_{2}-h_{1}\right|^{2} \mathrm{~d} x+C_{\varepsilon} \int_{0}^{b}\left|h_{2}^{\prime \prime}-h_{1}^{\prime \prime}\right|^{2} \mathrm{~d} x \leq \eta \int_{0}^{b}\left|h_{2}^{\prime \prime \prime}-h_{1}^{\prime \prime \prime}\right|^{2} \mathrm{~d} x+\eta \int_{0}^{b}\left|h_{2}^{\prime \prime}-h_{1}^{\prime \prime}\right|^{2} \mathrm{~d} x \\
& +C_{\eta}\left(1+\left\|h_{1}^{\prime \prime}\right\|_{\infty}^{2}+\left\|h_{2}^{\prime \prime}\right\|_{\infty}^{2}\right) \int_{0}^{b}\left|h_{2}^{\prime}-h_{1}^{\prime}\right|^{2} \mathrm{~d} x+C_{\eta}(1+D) \int_{0}^{b}\left|h_{2}-h_{1}\right|^{2} \mathrm{~d} x,
\end{aligned}
$$

for a small $\eta>0$ and for a function $D$ defined in $(0, \mathrm{~T})$ by

$$
D(t):=\sum_{i=1,2}\left(\left\|h_{i}^{\prime \prime}(\cdot, t)\right\|_{\infty}^{2}+\left\|h_{i}^{\prime \prime}(\cdot, t)\right\|_{\infty}^{4}+\left\|h_{i}^{\prime \prime \prime}(\cdot, t)\right\|_{\infty}^{2}\right)
$$

Step 3: We now insert (2.15) for $h_{1}$ and $h_{2}$ in (4.4). Since

$$
\left(\frac{h_{i}^{\prime \prime}}{J_{i}^{5}}\right)^{\prime \prime}=\left(\frac{h_{i}^{\prime \prime \prime}}{J_{i}^{5}}\right)^{\prime}-5\left(\frac{\left(h_{i}^{\prime \prime}\right)^{2} h_{i}^{\prime}}{J_{i}^{7}}\right)^{\prime}
$$

for $i=1,2$, integrating by parts and using the periodicity of $h_{1}(\cdot, t)$ and $h_{2}(\cdot, t)$, we have that

$$
\begin{aligned}
\frac{1}{2} \frac{\partial}{\partial t} \int_{0}^{b}\left|h_{2}^{\prime}-h_{1}^{\prime}\right|^{2} \mathrm{~d} x= & -\int_{0}^{b}\left[\varepsilon \frac{h_{2}^{\prime \prime \prime}}{J_{2}^{5}}-\frac{5 \varepsilon}{2} \frac{\left(h_{2}^{\prime \prime}\right)^{2} h_{2}^{\prime}}{J_{2}^{7}}+\partial_{1} \psi\left(-h_{2}^{\prime}, 1\right)\right]\left(J_{2}\left(h_{2}^{\prime \prime}-h_{1}^{\prime \prime}\right)\right)^{\prime} \mathrm{d} x \\
& +\int_{0}^{b}\left[\varepsilon \frac{h_{1}^{\prime \prime \prime}}{J_{1}^{5}}-\frac{5 \varepsilon}{2} \frac{\left(h_{1}^{\prime \prime}\right)^{2} h_{1}^{\prime}}{J_{1}^{7}}+\partial_{1} \psi\left(-h_{1}^{\prime}, 1\right)\right]\left(J_{1}\left(h_{2}^{\prime \prime}-h_{1}^{\prime \prime}\right)\right)^{\prime} \mathrm{d} x \\
& +\int_{0}^{b}\left(W_{2} J_{2}-W_{1} J_{1}\right)\left(h_{2}^{\prime \prime}-h_{1}^{\prime \prime}\right) \mathrm{d} x:=\bar{I}_{1}+\bar{I}_{2}+\bar{I}_{3} .
\end{aligned}
$$

Proceeding analogously to the second step, we estimate the integrals on the right-hand side of the previous equality. 
First, we observe that

$$
\begin{aligned}
\bar{I}_{1}+\bar{I}_{2}+\varepsilon \int_{0}^{b} \frac{\left|h_{2}^{\prime \prime \prime}-h_{1}^{\prime \prime \prime}\right|^{2}}{J_{2}^{4}} \mathrm{~d} x= & -\varepsilon \int_{0}^{b} h_{1}^{\prime \prime \prime}\left(\frac{1}{J_{2}^{4}}-\frac{1}{J_{1}^{4}}\right)\left(h_{2}^{\prime \prime \prime}-h_{1}^{\prime \prime \prime}\right) \mathrm{d} x \\
& -\varepsilon \int_{0}^{b}\left(\frac{h_{2}^{\prime \prime \prime} h_{2}^{\prime \prime} h_{2}^{\prime}}{J_{2}^{6}}-\frac{h_{1}^{\prime \prime \prime} h_{1}^{\prime \prime} h_{1}^{\prime}}{J_{1}^{6}}\right)\left(h_{2}^{\prime \prime}-h_{1}^{\prime \prime}\right) \mathrm{d} x \\
& +\frac{5 \varepsilon}{2} \int_{0}^{b}\left(\frac{\left(h_{2}^{\prime \prime}\right)^{2} h_{2}^{\prime}}{J_{2}^{6}}-\frac{\left(h_{1}^{\prime \prime}\right)^{2} h_{1}^{\prime}}{J_{1}^{6}}\right)\left(h_{2}^{\prime \prime \prime}-h_{1}^{\prime \prime \prime}\right) \mathrm{d} x \\
& +\frac{5 \varepsilon}{2} \int_{0}^{b}\left(\frac{\left(h_{2}^{\prime \prime}\right)^{3}\left(h_{2}^{\prime}\right)^{2}}{J_{2}^{8}}-\frac{\left(h_{1}^{\prime \prime}\right)^{3}\left(h_{1}^{\prime}\right)^{2}}{J_{1}^{8}}\right)\left(h_{2}^{\prime \prime}-h_{1}^{\prime \prime}\right) \mathrm{d} x \\
& -\int_{0}^{b}\left(\partial_{1} \psi\left(-h_{2}^{\prime}, 1\right) J_{2}-\partial_{1} \psi\left(-h_{1}^{\prime}, 1\right) J_{1}\right)\left(h_{2}^{\prime \prime \prime}-h_{1}^{\prime \prime \prime}\right) \mathrm{d} x \\
& -\int_{0}^{b}\left(\partial_{1} \psi\left(-h_{2}^{\prime}, 1\right) \frac{h_{2}^{\prime \prime} h_{2}^{\prime}}{J_{2}}-\partial_{1} \psi\left(-h_{1}^{\prime}, 1\right) \frac{h_{1}^{\prime \prime} h_{1}^{\prime}}{J_{1}}\right)\left(h_{2}^{\prime \prime}-h_{1}^{\prime \prime}\right) \mathrm{d} x .
\end{aligned}
$$

Thus, recalling (4.7) and using as before the facts that $h_{1}^{\prime}$ and $h_{2}^{\prime}$ are uniformly bounded, that for $n \in \mathbb{N}$, (4.8) holds, and that the functions $s \mapsto\left(1+s^{2}\right)^{-\frac{n}{2}}$ and $s \mapsto \partial_{1} \psi(s, 1)$ are locally Lipschitz continuous, we obtain

$$
\begin{aligned}
\bar{I}_{1}+ & \bar{I}_{2}+C_{\varepsilon} \int_{0}^{b}\left|h_{2}^{\prime \prime \prime}-h_{1}^{\prime \prime \prime}\right|^{2} \mathrm{~d} x \leq C\left[\int_{0}^{b}\left|h_{1}^{\prime \prime \prime}\right|\left|h_{2}^{\prime \prime \prime}-h_{1}^{\prime \prime \prime}\right|\left|h_{2}^{\prime}-h_{1}^{\prime}\right| \mathrm{d} x\right. \\
& +\int_{0}^{b}\left(\left|h_{2}^{\prime \prime}\right|\left|h_{2}^{\prime \prime}-h_{1}^{\prime \prime}\right|\right)\left(\left|h_{2}^{\prime \prime \prime}\right|\left|h_{2}^{\prime}-h_{1}^{\prime}\right|\right) \mathrm{d} x+\int_{0}^{b}\left|h_{2}^{\prime \prime}\right|\left|h_{2}^{\prime \prime \prime}-h_{1}^{\prime \prime \prime}\right|\left|h_{2}^{\prime \prime}-h_{1}^{\prime \prime}\right| \mathrm{d} x+\int_{0}^{b}\left|h_{1}^{\prime \prime \prime}\right|\left|h_{2}^{\prime \prime}-h_{1}^{\prime \prime}\right|^{2} \mathrm{~d} x \\
& +\int_{0}^{b}\left|h_{2}^{\prime \prime}\right|^{2}\left|h_{2}^{\prime \prime \prime}-h_{1}^{\prime \prime \prime}\right|\left|h_{2}^{\prime}-h_{1}^{\prime}\right| \mathrm{d} x+\left(\left\|h_{1}^{\prime \prime}\right\|_{\infty}+\left\|h_{2}^{\prime \prime}\right\|_{\infty}\right) \int_{0}^{b}\left|h_{2}^{\prime \prime \prime}-h_{1}^{\prime \prime \prime}\right|\left|h_{2}^{\prime \prime}-h_{1}^{\prime \prime}\right| \mathrm{d} x \\
& +\int_{0}^{b}\left(\left|h_{2}^{\prime \prime}\right|\left|h_{2}^{\prime \prime}-h_{1}^{\prime \prime}\right|\right)\left(\left|h_{2}^{\prime \prime}\right|^{2}\left|h_{2}^{\prime}-h_{1}^{\prime}\right|\right) \mathrm{d} x+\left(\left\|h_{1}^{\prime \prime}\right\|_{\infty}^{2}+\left\|h_{2}^{\prime \prime}\right\|_{\infty}^{2}\right) \int_{0}^{b}\left|h_{2}^{\prime \prime}-h_{1}^{\prime \prime}\right|^{2} \mathrm{~d} x \\
& \left.+\int_{0}^{b}\left|h_{2}^{\prime \prime \prime}-h_{1}^{\prime \prime \prime}\right|\left|h_{2}^{\prime}-h_{1}^{\prime}\right| \mathrm{d} x+\int_{0}^{b}\left|h_{2}^{\prime \prime}\right|\left|h_{2}^{\prime \prime}-h_{1}^{\prime \prime}\right|\left|h_{2}^{\prime}-h_{1}^{\prime}\right| \mathrm{d} x+\int_{0}^{b}\left|h_{2}^{\prime \prime}-h_{1}^{\prime \prime}\right|^{2} \mathrm{~d} x\right] .
\end{aligned}
$$

We then apply Young's inequality to each integral on the right-hand side of the previous inequality. Precisely, for the integrals that present the term $\left|h_{2}^{\prime \prime \prime}-h_{1}^{\prime \prime \prime}\right|$ we apply Young's inequality with a parameter $\eta>0$. In this way, we have

$$
\begin{aligned}
\bar{I}_{1}+\bar{I}_{2}+ & C_{\varepsilon} \int_{0}^{b}\left|h_{2}^{\prime \prime \prime}-h_{1}^{\prime \prime \prime}\right|^{2} \mathrm{~d} x \leq \eta \int_{0}^{b}\left|h_{2}^{\prime \prime \prime}-h_{1}^{\prime \prime \prime}\right|^{2} \mathrm{~d} x \\
& +C_{\eta}\left(1+\left\|h_{1}^{\prime \prime}\right\|_{\infty}^{2}+\left\|h_{2}^{\prime \prime}\right\|_{\infty}^{2}+\left\|h_{1}^{\prime \prime \prime}\right\|_{\infty}\right) \int_{0}^{b}\left|h_{2}^{\prime \prime}-h_{1}^{\prime \prime}\right|^{2} \mathrm{~d} x \\
& +C_{\eta}\left(1+\left\|h_{2}^{\prime \prime}\right\|_{\infty}^{2}+\left\|h_{2}^{\prime \prime}\right\|_{\infty}^{4}+\left\|h_{1}^{\prime \prime \prime}\right\|_{\infty}^{2}+\left\|h_{2}^{\prime \prime \prime}\right\|_{\infty}^{2}\right) \int_{0}^{b}\left|h_{2}^{\prime}-h_{1}^{\prime}\right|^{2} \mathrm{~d} x .
\end{aligned}
$$


Next, we estimate $\bar{I}_{3}$ from above. From the facts that the function $s \mapsto\left(1+s^{2}\right)^{\frac{1}{2}}$ is locally Lipschitz continuous, that $W_{i}$ and $h_{i}^{\prime}$ are uniformly bounded for $i=1,2$ and (4.11), it follows that

$$
\begin{aligned}
\bar{I}_{3} & \leq C \int_{0}^{b}\left|W_{2}-W_{1}\right|\left|h_{2}^{\prime \prime}-h_{1}^{\prime \prime}\right| \mathrm{d} x+C \int_{0}^{b}\left|h_{2}^{\prime \prime}-h_{1}^{\prime \prime}\right|\left|h_{2}^{\prime}-h_{1}^{\prime}\right| \mathrm{d} x \\
& \leq C \int_{0}^{b}\left|h_{2}^{\prime \prime}-h_{1}^{\prime \prime}\right|^{2} \mathrm{~d} x+C \int_{0}^{b}\left|h_{2}^{\prime}-h_{1}^{\prime}\right|^{2} \mathrm{~d} x+C \int_{0}^{b}\left|h_{2}-h_{1}\right|^{2} \mathrm{~d} x
\end{aligned}
$$

where we used Young's inequality and (4.11).

Now, since

$$
\left\|h_{2}^{\prime \prime}-h_{1}^{\prime \prime}\right\|_{L^{2}} \leq C\left\|h_{2}^{\prime \prime \prime}-h_{1}^{\prime \prime \prime}\right\|_{L^{2}}^{\frac{1}{2}}\left\|h_{2}^{\prime}-h_{1}^{\prime}\right\|_{L^{2}}^{\frac{1}{2}}
$$

by (5.6) applied to $h_{2}^{\prime}-h_{1}^{\prime}$ with $j=1$ and $m=2$, we observe that

$$
\begin{aligned}
C_{\eta}\left(1+\left\|h_{1}^{\prime \prime}\right\|_{\infty}^{2}\right. & \left.+\left\|h_{2}^{\prime \prime}\right\|_{\infty}^{2}+\left\|h_{1}^{\prime \prime \prime}\right\|_{\infty}\right) \int_{0}^{b}\left|h_{2}^{\prime \prime}-h_{1}^{\prime \prime}\right|^{2} \mathrm{~d} x \\
& \leq C_{\eta}\left(1+\left\|h_{1}^{\prime \prime}\right\|_{\infty}^{2}+\left\|h_{2}^{\prime \prime}\right\|_{\infty}^{2}+\left\|h_{1}^{\prime \prime \prime}\right\|_{\infty}\right)\left\|h_{2}^{\prime \prime \prime}-h_{1}^{\prime \prime \prime}\right\|_{L^{2}}\left\|h_{2}^{\prime}-h_{1}^{\prime}\right\|_{L^{2}} \\
& \leq \eta \int_{0}^{b}\left|h_{2}^{\prime \prime \prime}-h_{1}^{\prime \prime \prime}\right|^{2}+C_{\eta}\left(1+\left\|h_{1}^{\prime \prime}\right\|_{\infty}^{4}+\left\|h_{2}^{\prime \prime}\right\|_{\infty}^{4}+\left\|h_{1}^{\prime \prime \prime}\right\|_{\infty}^{2}\right) \int_{0}^{b}\left|h_{2}^{\prime}-h_{1}^{\prime}\right|^{2}
\end{aligned}
$$

where, in the last inequality, we used again Young's inequality for $\eta>0$.

Finally, by (4.15), (4.16), (4.17) and (4.18), we obtain

$$
\begin{aligned}
& \frac{\partial}{\partial t} \int_{0}^{b}\left|h_{2}^{\prime}-h_{1}^{\prime}\right|^{2} \mathrm{~d} x+C_{\varepsilon} \int_{0}^{b}\left|h_{2}^{\prime \prime \prime}-h_{1}^{\prime \prime \prime}\right|^{2} \mathrm{~d} x \leq \\
& \quad \leq \eta \int_{0}^{b}\left|h_{2}^{\prime \prime \prime}-h_{1}^{\prime \prime \prime}\right|^{2} \mathrm{~d} x+C_{\eta}(1+D) \int_{0}^{b}\left|h_{2}^{\prime}-h_{1}^{\prime}\right|^{2} \mathrm{~d} x+C \int_{0}^{b}\left|h_{2}-h_{1}\right|^{2} \mathrm{~d} x,
\end{aligned}
$$

where $D$ is the function defined in $(0, T)$ by (4.14).

Step 4: Adding (4.13) and (4.19), and choosing $\eta$ small enough, we deduce that

$$
\frac{\partial H}{\partial t}(t) \leq C(1+D(t)) H(t)
$$

for some costant $C>0$ and for each $t \in(0, T)$. We note that, for each $t \in(0, T)$ and for $i=1,2$, by (5.7) with $m=2, p=2$, and $q=\infty$ applied to $h_{i}^{\prime \prime}(\cdot, t)$, we have

$$
\left\|h_{i}^{\prime \prime}(\cdot, t)\right\|_{\infty} \leq C\left\|h_{i}^{(\mathrm{iv})}(\cdot, t)\right\|_{L^{2}(0, b)}^{\frac{1}{4}}\left\|h_{i}^{\prime \prime}(\cdot, t)\right\|_{L^{2}(0, b)}^{\frac{3}{4}} \leq C M^{\frac{3}{4}}\left\|h_{i}^{(\mathrm{iv})}(\cdot, t)\right\|_{L^{2}(0, b)}^{\frac{1}{4}},
$$

and by (5.8) with $m=2, j=1, p=2$, and $q=\infty$ again applied to $h_{i}^{\prime \prime}(\cdot, t)$, we have

$$
\left\|h_{i}^{\prime \prime \prime}(\cdot, t)\right\|_{\infty} \leq C\left\|h_{i}^{(\mathrm{iv})}(\cdot, t)\right\|_{L^{2}(0, b)}^{\frac{3}{4}}\left\|h_{i}^{\prime \prime}(\cdot, t)\right\|_{L^{2}(0, b)}^{\frac{1}{4}} \leq C M^{\frac{1}{4}}\left\|h_{i}^{(\mathrm{iv})}(\cdot, t)\right\|_{L^{2}(0, b)}^{\frac{3}{4}} .
$$

Therefore, we may find a constant $C>0$ that depends only on $M$ such that $D(t) \leq C G(t)$, and so (4.2) follows from (4.20). In view of the fact that $G \in L^{1}(0, T)$, we may apply 
Gronwall's Lemma to obtain that $H$ satisfies

$$
H(t) \leq H(0) \exp \left(\int_{0}^{t} G(s) \mathrm{d} s\right)
$$

for every $t \in[0, T]$. Since $H(0)=0$, this concludes the proof.

\section{APPENDIX}

In this section we collect some results used throughout the paper. We begin by establishing a Korn-type inequality for subgraphs of Lipschitz functions.

Lemma 5.1. Let $h:[0, b] \rightarrow[-L, L]$ be a Lipschitz function with $\operatorname{Lip} h \leq L$ for some $L>0$ and consider $U_{h}:=\{z=(x, y): 0<x<b,-L(1+3 b)<y<h(x)\}$. If $1<p<\infty$, then there exists a constant $C=C(p, b, L)>0$ such that

$$
\int_{U_{h}}|u|^{p} \mathrm{~d} z+\int_{U_{h}}|\nabla u|^{p} \mathrm{~d} z \leq C \int_{U_{h}}|E(u)|^{p} \mathrm{~d} z
$$

for all $u \in W^{1, p}\left(U_{h} ; \mathbb{R}^{2}\right)$ with $u(\cdot,-L(1+3 b))=0$ (in the sense of traces).

Proof. Fix a ball $B$ contained in $(0, b) \times(-L(1+3 b),-L(1+2 b))$. Since $U_{h}$ is an open bounded domain starshaped with respect to $B$, by a classical version of Korn's inequality (see $[32,36])$ there exists a constant $C_{1}=C_{1}(p, b, L)>0$ such that

$$
\int_{U_{h}}|\nabla u|^{p} \mathrm{~d} z \leq C_{1}\left(\int_{U_{h}}|u|^{p} \mathrm{~d} z+\int_{U_{h}}|E(u)|^{p} \mathrm{~d} z\right)
$$

for all $u \in W^{1, p}\left(U_{h} ; \mathbb{R}^{2}\right)$. Thus, it is enough to prove that

$$
\int_{U_{h}}|u|^{p} \mathrm{~d} z \leq C_{2} \int_{U_{h}}|E(u)|^{p} \mathrm{~d} z
$$

for some constant $C_{2}=C_{2}(p, b, L)>0$. By contradiction, assume that there exists a sequence $\left\{h_{n}\right\}$ as in the statement and a sequence $\left\{u_{n}\right\} \subset W^{1, p}\left(U_{h_{n}} ; \mathbb{R}^{2}\right)$ of functions with $u_{n}(\cdot,-L(1+3 b))=0$ (in the sense of traces) such that

$$
\int_{U_{h_{n}}}\left|u_{n}\right|^{p} \mathrm{~d} z>n \int_{U_{h_{n}}}\left|E\left(u_{n}\right)\right|^{p} \mathrm{~d} z .
$$

By the Ascoli-Arzelà Theorem, since $\left\{h_{n}\right\}$ is bounded in $C^{0,1}([0, b])$ by $L$, up to a subsequence (not relabeled), it converges uniformly to a Lipschitz function $\bar{h}$ with $\operatorname{Lip} \bar{h} \leq L$. Furthermore, for every $n \in \mathbb{N}$, the function

satisfies

$$
v_{n}:=\frac{u_{n}}{\left\|u_{n}\right\|_{L^{p}\left(U_{h_{n}}\right)}}
$$

$$
\int_{U_{h_{n}}}\left|v_{n}\right|^{p} \mathrm{~d} z=1, \quad \int_{U_{h_{n}}}\left|E\left(v_{n}\right)\right|^{p} \mathrm{~d} z \rightarrow 0 \text { as } n \rightarrow \infty,
$$


and its trace on the segment $(0, b) \times\{-L(1+3 b)\}$ is equal to zero. Hence,

$$
\sup _{n} \int_{U_{h_{n}}}\left|\nabla v_{n}\right|^{p} \mathrm{~d} z<+\infty
$$

by (5.2), and since $U_{h_{n}}$ has Lipschitz boundary we can extend the functions $v_{n}$ to the rectangle $R_{L}:=(0, b) \times(L(1+3 b),-L(1+3 b))$ in such a way that $\left\{v_{n}\right\}$ is bounded in $W^{1, p}\left(R_{L} ; \mathbb{R}^{2}\right)$ with null trace on $(0, b) \times\{-L(1+3 b)\}$. Thus, up to a subsequence (not relabeled), $\left\{v_{n}\right\}$ converges weakly in $W^{1, p}\left(R_{L} ; \mathbb{R}^{2}\right)$ to some function $v$. Note that $(5.4)$ implies that

$$
\int_{U_{\bar{h}}}|v|^{p} \mathrm{~d} z=1
$$

since $\left\{v_{n} \chi_{U_{h_{n}}}\right\}$ converges to $v \chi_{U_{\bar{h}}}$ in $L^{p}\left(R_{L} ; \mathbb{R}^{2}\right)$ by the Lebesgue Dominated Theorem and the uniqueness of the limit. Moreover, $v$ has trace zero on the segment $(0, b) \times\{-L(1+3 b)\}$ (see [29]), and $\left\{E\left(v_{n}\right)\right\}$ converges weakly to $E(v)$ in $L^{p}\left(R_{L} ; \mathbb{R}^{2}\right)$. Thus, in view of the uniform convergence of $\left\{h_{n}\right\}$ to $\bar{h}$ and by the Lebesgue Monotone Convergence Theorem, we have

$$
\int_{U_{\bar{h}}}|E(v)|^{p} \mathrm{~d} z \leq \liminf _{n \rightarrow \infty} \int_{U_{h_{n}}}\left|E\left(v_{n}\right)\right|^{p} \mathrm{~d} z=0,
$$

and so $E(v) \equiv 0 \mathcal{L}^{2}$-a.e in $U_{\bar{h}}$. Since $U_{\bar{h}}$ is connected, this yields that $v(z)=a+A z$ for some $a \in \mathbb{R}^{2}$ and some skew-symmetric matrix $A \in \mathbb{M}^{2 \times 2}$. Thus, since $v$ is continuous, $v(\cdot,-L(1+3 b))=0$ in $(0, b)$ and so $a=0$ and $A=0$. We have reached a contradiction with (5.5).

The following two lemmas provide the identities used to derive (2.15). The proofs can be found in the Appendix of [19].

Lemma 5.2. Let $g$ be the function introduced in (2.2). Then,

$$
g(\theta)+g_{\theta \theta}(\theta)=\frac{\partial_{11} \psi(\cos \theta, \sin \theta)}{\sin ^{2} \theta}
$$

for every $\theta \in(0,2 \pi) \backslash\{\pi\}$.

Lemma 5.3. The curvature regularization term satisfies the identity

$$
k_{\sigma \sigma}+\frac{1}{2} k^{3}=\left(\frac{h_{x x}}{J^{5}}\right)_{x x}+\frac{5}{2}\left(\frac{h_{x x}^{2}}{J^{7}} h_{x}\right)_{x}
$$

for h sufficiently smooth.

For the convenience of the reader, we present here some interpolation inequalities that are used throughout the paper, and that are essentially contained in [1] and in the Appendix of [19] (see also [30]). We recall that given a bounded open interval $I \subset \mathbb{R}, W_{\#}^{m, p}(I)$ denotes the space of all functions in $W_{\mathrm{loc}}^{m, p}(\mathbb{R})$ that are $|I|$-periodic, endowed with the norm of $W^{m, p}(I)$. 
Theorem 5.4. Let $I \subset \mathbb{R}$ be a bounded open interval. Let $j, m$ be positive integers such that $0 \leq j<m$, and let $1 \leq p \leq q \leq \infty$ be such that $m p>1$. Then, there exists a constant $K>0$ such that for all $f \in W_{\#}^{m, p}(I)$

$$
\left\|f^{(j)}\right\|_{L^{p}(I)} \leq K\left\|f^{(m)}\right\|_{L^{p}(I)}^{\frac{j}{m}}\|f\|_{L^{p}(I)}^{\frac{m-j}{m}} .
$$

In addition, if either $f$ vanishes at the boundary or $\int_{I} f \mathrm{~d} x=0$, then

$$
\|f\|_{L^{q}(I)} \leq K\left\|f^{(m)}\right\|_{L^{p}(I)}^{\theta}\|f\|_{L^{p}(I)}^{1-\theta},
$$

where $\theta:=\frac{1}{m}\left(\frac{1}{p}-\frac{1}{q}\right)$.

The following result follows from Theorem 5.4.

Corollary 5.5. Let $I \subset \mathbb{R}$ be a bounded open interval. Let $j, m$ be positive integers such that $0<j<m$ and let $1 \leq p \leq q \leq \infty$ be such that $(m-j) p>1$. Then, there exists a constant $K>0$ such that for all $f \in W_{\#}^{m, p}(I)$

$$
\left\|f^{(j)}\right\|_{L^{q}(I)} \leq K\left\|f^{(m)}\right\|_{L^{p}(I)}^{\eta}\|f\|_{L^{p}(I)}^{1-\eta},
$$

where $\eta:=\frac{1}{m}\left(\frac{1}{p}-\frac{1}{q}+j\right)$.

Proof. Since $f^{(j)} \in W_{\#}^{m-j, p}(I)$ and $\int_{I} f^{(j)} \mathrm{d} x=0$, by (5.7) we have

$$
\left\|f^{(j)}\right\|_{L^{q}(I)} \leq K\left\|f^{(m)}\right\|_{L^{p}(I)}^{\theta}\left\|f^{(j)}\right\|_{L^{p}(I)}^{1-\theta}
$$

with $\theta:=\frac{1}{m-j}\left(\frac{1}{p}-\frac{1}{q}\right)$, which, together with (5.6), yields (5.8).

Finally, the following elliptic estimate was established in [19, Lemma 6.10] using [21, Proposition 8.9]. Recall Remark 2.5 for the notation.

Lemma 5.6. Let $M>0$ and $c_{0}>0$. Consider $h_{1}, h_{2} \in H_{\#}^{2}(0, b)$ with $h_{i} \geq c_{0}$ and $\left\|h_{i}\right\|_{H_{\#}^{2}(0, b)} \leq M$ for $i=1,2$, and let $u_{1}$ and $u_{2}$ the corresponding elastic equilibrium in $\Omega_{h_{1}}$ and $\Omega_{h_{2}}$, respectively. Then, for every $\alpha \in\left(0, \frac{1}{2}\right]$

$$
\left\|E\left(u_{1}\right)\left(\cdot, h_{1}(\cdot)\right)-E\left(u_{2}\right)\left(\cdot, h_{2}(\cdot)\right)\right\|_{C^{1, \alpha}([0, b])} \leq C\left\|h_{1}-h_{2}\right\|_{C^{1, \alpha}([0, b])}
$$

for some constant $C>0$ depending only on $M, c_{0}$ and $\alpha$.

\section{ACKNOWLEDGMENT}

This work is part of a thesis to be submitted by the author in partial fulfilment of the requirements for the degree of Doctor of Philosophy in Mathematics under the supervision of I. Fonseca and G. Leoni at Carnegie Mellon University. The author is indebted to his supervisors for suggesting this topic as well as for their guidance. Moreover, the research of P. Piovano was partially funded by the National Science Foundation under Grants No. DMS-0401763 and DMS-0905778 of I. Fonseca, and No. DMS-0708039 and DMS-1007989 of G. Leoni. The author also acknowledge support of the National Science Foundation 
under the PIRE Grant No. OISE-0967140. My thanks go also to N. Fusco and M. Morini, for their insightful discussions.

\section{REFERENCES}

[1] Adams R.A., Fournier J.F., Sobolev Spaces. Second edition. Pure and Applied Mathematics (Amsterdam), 140. Elsevier/Academic Press, Amsterdam, 2003.

[2] Almgren F., Taylor J., Wang L., Curvature-driven flows: a variational approach. SIAM J. Control Optim. 31 (1993), 387-438.

[3] Ambrosio L., Minimizing movements. Rend. Accad. Naz. Sci. XL Mem. Mat. Appl. 5 (1995), 191-246.

[4] Ambrosio L., Gigli N., Savaré G., Gradient Flows in Metric Spaces and in the Space of Probability Measures. Second edition. Lectures in Mathematics ETH Zürich. Birkhäuser Verlag, Basel, 2008.

[5] Angenent S., Parabolic equations for curves on surfaces. I. Curves with p-integrable curvature. Ann. of Math. 132 (1990), 451-483.

[6] Angenent S., Parabolic equations for curves on surfaces. II. Intersections, blow-up and generalized solutions. Ann. of Math. 133 (1991), 171-215.

[7] Angenent S., Gurtin M. E., Multiphase thermomechanics with interfacial structure. II. Evolution of an isothermal interface. Arch. Rational Mech. Anal. 108 (1989), 323-391.

[8] Bellettini G., Caselles V., Chambolle A., Novaga M., The volume preserving crystalline mean curvature flow of convex sets in $\mathbb{R}^{N}$.. J. Math. Pures Appl. 92 (2009), 499-527.

[9] Bellettini G., Mantegazza C., Novaga M., Singular perturbations of mean curvature flow. J. Differential Geom. 75 (2007), 403-431.

[10] Bonnetier E., Chambolle A., Computing the equilibrium configuration of epitaxially strained crystalline films. SIAM J. Appl. Math. 62 (2002), 1093-1121.

[11] Burger M., Hausser F., Stöcker C., Voigt A., A level set approach to anisotropic flows with curvature regularization. J. Comput. Phys. 225 (2007), 183-205.

[12] Cahn J. W., Taylor J. E., Overview N0. 113 - Surface motion by surface-diffusion. Acta Metall. Mater. 42 (1994), 1045-1063.

[13] Caselles V., Chambolle A., Anisotropic curvature-driven flow of convex sets. Nonlinear Anal. 65 (2006), 1547-1577.

[14] Di Carlo A., Gurtin M., Podio-Guidugli P., A regularized equation for anisotropic motion-bycurvature. SIAM J. Appl. Math 52 (1992), 1111-1119.

[15] Di Nezza E., Palatucci G., Valdinoci E., Hitchhikers guide to the fractional Sobolev spaces. Preprint 2011.

[16] Dondl P. W., Bhattacharya K., A sharp interface model for the propagation of martensitic phase boundaries. Arch. Ration. Mech. Anal. 197 (2010), 599-617.

[17] Eminenti M., Mantegazza C., Some properties of the distance function and a conjecture of De Giorgi. J. Geom. Anal. 14 (2004), 267-279.

[18] Fonseca I., Fusco N., LeOni G., Morini M., Equilibrium configurations of epitaxially strained crystalline films: existence and regularity results. Arch. Rational Mech. Anal. 186 (2007), 477-537.

[19] Fonseca I., Fusco N., Leoni G., Morini M., Motion of elastic thin films by anisotropic surface diffusion with curvature regularization. Arch. Rational Mech. Anal. DOI 10.1007/s00205-012-0509-4.

[20] Fried E., Gurtin M. E., A unified treatment of evolving interfaces accounting for small deformations and atomic transport with emphasis on grain-boundaries and epitaxy. Adv. Appl. Mech. 40 (2004), $1-177$.

[21] Fusco N., Morini M., Equilibrium configurations of epitaxially strained elastic films: second order minimality conditions and qualitative properties of solutions. Preprint 2009.

[22] Giusti E., Minimal Surfaces and Functions of Bounded Variation. Monographs in Mathematics. Vol. 80. Birkhäuser Verlag, Basel, 1984. 
[23] Gurtin M. E., Multiphase thermomechanics with interfacial structure. I. Heat conduction and the capillary balance law. Arch. Rational Mech. Anal. 104 (1988), 195-221.

[24] Gurtin M. E., Jabbour M. E., Interface evolution in three dimensions with curvature-dependent energy and surface diffusion: interface-controlled evolution, phase transitions, epitaxial growth of elastic films. Arch. Rational Mech. Anal. 163 (2002), 171-208.

[25] Gurtin M. E., Soner, H. M., Souganidis, P. E., Anisotropic motion of an interface relaxed by the formation of infinitesimal wrinkles. J. Differential Equations 119 (1995), 54-108.

[26] Gurtin M. E., Struthers A., Multiphase thermomechanics with interfacial structure. III. Evolving phase boundaries in the presence of bulk deformation. Arch. Rational Mech. Anal. 112 (1990), 97-160.

[27] Herring C., Surface Tension as a Motivation for Sintering. The physics of powder metallurgy, McGraw-Hill, New York, 1951.

[28] Herring C., Some theorems on the free energies of crystal surfaces. Physical Review 82 (1951), 87-93.

[29] Leoni G., A First Course in Sobolev Spaces. Graduate Studies in Mathematics. Vol. 105. Am. Math. Soc. Providence, Rhode Island, 2009.

[30] LEONI G., Interpolation for intermediate derivatives. http://www.ams.org/publications/authors/books /postpub/gsm-105

[31] Li B., Lowengrub J., Rätz A., Voigt A., Geometric evolution laws for thin crystalline films: modeling and numerics. Commun. Comput. Phys. 6 (2009), 433-482.

[32] Mosolov P.P., Muasnikov V.P., A proof of Korn's inequality. Dokl. Akad. Nauk SSSR 201 (1971), $37-39$.

[33] Mullins W. W., Two-dimensional motion of idealized grain boundaries. J. Appl. Phys. 27 (1956), 900-904.

[34] Mullins W. W., Theory of thermal grooving. J. Appl. Phys. 28 (1957), 333-339.

[35] Mastroberardino A., Spencer B. J., Three-dimensional equilibrium crystal shapes with corner energy regularization. IMA J. Appl. Math. 75 (2010), 190-205.

[36] Necas J., HlaváceK I., Mathematical Theory of Elastic and Elasto-Plastic Bodies: an Introduction. Studies in Applied Mechanics. Vol. 3. Elsevier Amsterdam-New York, 1980.

[37] Scianna G., Tilli P., A variational approach to the Hele-Shaw flow with injection. Comm. Partial Differential Equations 30 (2005), 1359-1378.

[38] Spencer B. J., Asymptotic solutions for the equilibrium crystal shape with small corner energy regularization. Phys. Rev. E 69 (2004), 011603.

[39] Stöcker C., Voigt A., A level set approach to anisotropic surface evolution with free adatoms. SIAM J. Appl. Math. 69 (2008), 64-80.

[40] Taylor J. E., Cahn J. W., Handwerker C. A., Overview No. 98 - Geometric models of crystal growth. Acta Metall. Mater. 40 (1992), 1443-1474.

Department of Mathematical Sciences, Carnegie Mellon University, Pittsburgh, PA 15213

E-mail address: ppiovano@andrew.cmu.edu 